\title{
miR-320a in serum exosomes promotes myocardial fibroblast proliferation via regulating the PIK3CA/Akt/mTOR signaling pathway in $\mathrm{HEH} 2$ cells
}

\author{
QING-GAO WANG ${ }^{1}$, BRIAN CHI-YAN CHENG ${ }^{2}$, YA-ZHOU HE $^{1}$, LI-JUAN LI $^{1}$, \\ YUN LING ${ }^{3}$, GAN LUO $^{4}$, LI WANG $^{4}$, SHAN LIANG ${ }^{1}$ and YI ZHANG ${ }^{4}$ \\ ${ }^{1}$ Department of Cardiology, First Affiliated Hospital, Guangxi University of Chinese Medicine, Nanning, \\ Guangxi Zhuang Autonomous Region 530023; ${ }^{2}$ College of Professional and Continuing Education, \\ The Hong Kong Polytechnic University, Hong Kong 999077, SAR; ${ }^{3}$ School of Nursing, \\ Guangxi University of Chinese Medicine, Nanning, Guangxi Zhuang Autonomous Region 530200; \\ ${ }^{4}$ Department of Pharmacology, School of Chinese Materia Medica, \\ Beijing University of Chinese Medicine, Beijing 102488, P.R. China
}

Received May 1, 2020; Accepted March 25, 2021

DOI: $10.3892 / \mathrm{etm} .2021 .10305$

\begin{abstract}
MicroRNAs (miRNAs/miRs) serve an important role in the pathogenesis of chronic heart failure $(\mathrm{CHF})$. A number of reports have illustrated the regulatory effect of serum exosomal miRNA on myocardial fibrosis. The present study aimed to investigate the expression of miR-320a in serum exosomes, as well as the effect of miR-320a on myocardial fibroblast proliferation. Serum exosome samples from 10 patients with $\mathrm{CHF}$ and 5 healthy volunteers were obtained and characterized. mRNA and protein expression levels were measured via reverse transcription-quantitative PCR and western blotting, respectively. The content of soluble growth stimulation expressed gene 2 (sST2) was determined via ELISA. HEH2 cell viability and apoptosis were detected by performing MTT assays and flow cytometry, respectively. The results demonstrated that serum miR-320a expression levels and sST2 content were significantly increased in patients with $\mathrm{CHF}$ compared with healthy controls, and the expression of serum miR-320a was significantly correlated
\end{abstract}

Correspondence to: Dr Yi Zhang, Department of Pharmacology, School of Chinese Materia Medica, Beijing University of Chinese Medicine, Li Ping Building B210, Sunshine South Street, Fang Shan Square, Beijing 102488, P.R. China

E-mail: yizhang714@163.com

Abbreviations: CHF, chronic heart failure; sST2, soluble growth stimulation expressed gene 2; GEO, Gene Expression Omnibus; HMDD, Human MicroRNA Disease Database; LVEF, left ventricular ejection fraction; NT-proBNP, N-Terminal pro-brain natriuretic peptide; NYHA, New York Heart Association; PIK3CA, phosphoinositide-3-kinase catalytic $\alpha$ polypeptide gene; $\alpha$-SMA, $\alpha$-smooth muscle actin

Key words: $\mathrm{CHF}$, exosome, HEH2 cells, myocardial fibrosis, PIK3CA/Akt/mTOR signaling pathway with clinical CHF indexes. miR-320a expression levels were significantly increased in exosomes isolated from patients with CHF compared with those isolated from healthy controls. Phosphoinositide-3-kinase catalytic $\alpha$ polypeptide gene (PIK3CA) expression levels and sST2 content were increased in $\mathrm{HEH} 2$ cells following transfection with miR-320a mimics compared with NC-mimic, whereas miR-320a inhibitor displayed contrasting effects by reduced the cell viability and apoptosis in myocardial fibroblasts compared with the NC-inhibitor group. The protein expression levels of collagen I, collagen III, $\alpha$-smooth muscle actin, phosphorylated (p)-mTOR (ser 2448)/mTOR, p-Akt (ser 473)/Akt, p-Akt (thr 308)/Akt and PIK3CA were significantly increased in miR-320a mimic-transfected HEH2 cells compared with the NC-mimics groups. By contrast, miR-320a inhibitor notably downregulated the expression levels of these proteins compared with the NC-inhibitor group. Collectively, the results of the present study demonstrated that miR-320a promoted myocardial fibroblast proliferation via regulating the PIK3CA/Akt/mTOR signaling pathway in HEH2 cells, suggesting that serum exosomal miR-320a may serve as a potential biomarker for the diagnosis of CHF.

\section{Introduction}

Chronic heart failure (CHF) is one of the leading causes of hospitalization and re-admissions in the elderly, accounting for a vast proportion of national healthcare expenditure in developed countries $(1,2)$. The pathophysiology of CHF is complex. Left ventricular remodeling serves an important role in the development of $\mathrm{CHF}$ (3), resulting in increases in the ventricular wall mass, heart chamber enlargement and a more spherical chamber conformation, which leads to a progressive decline in left ventricular performance (4). Among all the pathogenic processes of left ventricular remodeling, myocardial fibrosis is the key determinant, which is characterized by disordered collagen turnover and excessive diffuse collagen (including 
collagen type I and III) accumulation in the interstitial and perivascular spaces (5). Therefore, decreasing myocardial fibrosis may serve as an effective therapeutic strategy for CHF.

The PI3K/AKT/mTOR signaling pathway serves a pivotal role in the regulation of multiple biological processes, including cell proliferation, apoptosis, autophagy and metabolism $(6,7)$. Certain studies have also reported that PI3K/AKT/mTOR signaling regulates myocardial fibrosis following injury $(8,9)$. Moreover, TGF- $\beta$ induces the activation of pro-fibrotic gene endothelin-1, which initiates PI3K/Akt signaling (10). As the downstream protein of Akt, elevated mTOR activity induces excessive collagen production, including collagen I, collagen III and $\alpha$-smooth muscle actin (SMA), leading to excessive scarring and fibrosis (11).

MicroRNAs (miRNAs/miRs) are a class of short, highly conserved, non-coding RNA fragments that are involved in the regulation of gene expression at the post-transcriptional level via inhibiting their translation (12). miRNAs regulate a wide array of biological processes, including cell differentiation, apoptosis, inflammation and metabolism, and are involved in the pathogenesis of numerous diseases, including cancer (13-15). Previous studies have reported that miRNAs may serve an important role in regulating myocardial fibrosis, with circulating miRNAs emerging as promising biomarkers $(16,17)$. Therefore, the association between miRNAs and myocardial fibrosis has become an area of intensive research.

Exosomes are membrane-bound extracellular vesicles (size, 30-200 nm) that can carry biological information and transmit signals and molecules to target cells $(18,19)$. In recent years, it has been reported that miRNAs could be carried and transferred by exosomes from donor to recipient cells via target cell membrane fusion (20). This exosome-mediated process can regulate target gene expression, which serves essential roles in numerous human diseases, including cancer (21), Alzheimer's disease (22) and CHF (23). Notably, miR-320a has attracted increasing attention in cardiovascular biology $(24,25)$. However, the expression and potential roles of miR-320a, particularly for miR-320a from serum exosomes, in myocardial fibrosis are not completely understood. To the best of our knowledge, the present study was the first to investigate the potential use of exosomal miR-320a as a biomarker for myocardial fibrosis in patients with CHF. The present study also assessed the effect of miR-320a on myocardial fibroblast proliferation, as well as the potential underlying molecular mechanisms.

\section{Materials and methods}

Reagents and antibodies. The MTT and PKH67 Green Fluorescent Cell Linker kits were obtained from Sigma-Aldrich (Merck KGaA). FBS and penicillin-streptomycin solution were purchased from HyClone (Cytiva). DMEM was obtained from Gibco (Thermo Fisher Scientific, Inc.). The human soluble growth stimulation expressed gene 2 (sST2) ELISA kit (cat. no. ml060123) was obtained from ML Bio Solutions. The miRcute miRNA First-Strand cDNA Synthesis kit (cat. no. KR201) and the miRcute miRNA qPCR Detection kit (SYBR-Green, cat. no. FP401) were purchased from Tiangen Biotech Co., Ltd. Primary antibodies targeted against tumor susceptibility 101 (TSG101; cat. no. ab125011), CD63 (cat. no. ab134045), CD9 (cat. no. ab223052), Collagen I (cat. no. ab6308), Collagen III (cat. no. ab6310), $\alpha$-SMA (cat. no. ab32575) and phosphorylated (p)-Akt (ser 473; cat. no. ab194201) were purchased from Abcam. The anti-Akt primary antibody (cat. no. AF6261) was purchased from Affinity Biosciences. The HRP-conjugated goat anti-rabbit IgG secondary antibody (cat. no. SA00001-2) and anti- $\beta$-actin primary antibody (cat. no. 20536-1-AP) were obtained from ProteinTech Group Inc. The primary antibodies targeted against p-mTOR (Ser2481; cat. no. 2974), p-mTOR (Ser2448; cat. no. 2971), mTOR (cat. no. 2972) and p-Akt (Thr308; cat. no. 9275) were obtained from Cell Signaling Technology, Inc. The anti-phosphoinositide-3-kinase catalytic $\alpha$ polypeptide gene (PIK3CA) primary antibody (cat. no. PB0351) was obtained from Boster Biological Technology. The anti-GAPDH primary antibody (TA-08) was bought from OriGene Technologies, Inc. miR-320a, forward, 5'-AAAAGC TGGGTTGAGAGGGCGA-3' and the Universal primer as a reverse; PIK3CA, forward, 5'-GACTGTGTGGGACTTATT GAGG-3' and reverse, 5'-TGATGTAGTGTGTGGCTGTTG A-3'; GAPDH, forward, 5'-GGTGTGAACCATGAGAAG TATGA-3' and reverse, 5'-GAGTCCTTCCACGATACCAAA G-3'; and U6, forward, 5'-CTCGCTTCGGCAGCACA-3' and reverse, 5'-AACGCTTCACGAATTTGCGT-3' were synthesized by Shanghai Gene Pharma Co., Ltd. Lipofectamine ${ }^{\circledR}$ 2000 transfection reagent (cat. no. 11668027) was purchased from Thermo Fisher Scientific, Inc.

Inclusion and exclusion criteria of the patients and healthy volunteers. A total of 10 patients with CHF (mean age, 64.6 years; age range, 51-73 years; 7 male patients and 3 female patients) and 5 healthy volunteers (mean age, 56.6 years; age range, 51-62 years; 3 male volunteers and 2 female volunteers) were recruited at the First Affiliated Hospital of Guangxi University of Chinese Medicine (Guangxi Zhuang Autonomous Region; P.R. China). The date range of recruitment from June 2017 to June 2017. The inclusion criteria of the patients with patients were based on the 2014 Chinese CHF Diagnosis and Treatment guidelines (26). The exclusion criteria of the CHF patients included: i) Pregnancy; ii) received treatment with chemotherapeutic agents; and iii) diagnosed with any other diseases, including primary vascular heart diseases, congenital heart diseases, rheumatic diseases and tumors.

Healthy volunteers had no coronary disease, vascular disease or evidence of cardiomyopathy. In patients with CHF, left ventricular ejection fraction (LVEF) and left ventricular end-diastolic diameter (LVEDD) were assessed using Color Doppler Ultrasonic Diagnostic Equipment (General Electric). New York Heart Association (NYHA) classification of patients (27) with CHF was based on the patient's limitations during physical activity according to the 2014 Chinese CHF Diagnosis and Treatment guidelines. Serum levels of NT-proB-type natriuretic peptide (NT-proBNP) were determined with a commercially available electrochemiluminescence immunoassay (cat. no. 08836736190; Roche Diagnostics) according to the manufacturer's protocol.

The present study was approved by the Institutional Review Board of the First Affiliated Hospital of Guang Xi University of Chinese Medicine [approval no. (2015)012]. All participants provided written informed consent. 
Serum sample collection. Upon admission to the hospital, $\sim 10 \mathrm{ml}$ venous blood samples were obtained from healthy volunteers and patients with CHF. Serum samples were collected using pro-coagulation tubes, allowed to stand for $2 \mathrm{~h}$ and then centrifuged at 3,000 x g for $10 \mathrm{~min}$ at room temperature. At least $4 \mathrm{ml}$ serum was collected from each sample and stored at $-20^{\circ} \mathrm{C}$.

Exosome isolation and particle size identification. Serum was centrifuged at $2,000 \mathrm{x} \mathrm{g}$ for $20 \mathrm{~min}$ at $4^{\circ} \mathrm{C}$ in order to sediment debris. Subsequently, an exoRNeasy Serum-Plasma Midi kit (Qiagen $\mathrm{GmbH}$ ) was used according to the manufacturer's protocol. Briefly, $4 \mathrm{ml}$ serum for each group and XBP reagent were added into the separation column and centrifuged at $500 \mathrm{x} \mathrm{g}$ for $1 \mathrm{~min}$ at room temperature, and the supernatant was collected as exosome residues. Subsequently, $10 \mathrm{ml} \mathrm{XWP}$ reagent was added into the column and centrifuged at $500 \mathrm{xg}$ for $5 \mathrm{~min}$ at room temperature, and the supernatant was discarded. Finally, $1 \mathrm{ml} \mathrm{XE}$ reagent was added into the column and exosomes were collected by centrifugation at $5,000 \mathrm{x} \mathrm{g}$ for $5 \mathrm{~min}$ at room temperature. The size of the exosomes was quantified using ZetaView PMX110 Nanoparticle Size Analyzer (Particle Metrix $\mathrm{GmbH}$ ) and the corresponding software (ZetaView PMX 110, version 8.04.02).

Transmission electron microscopy. The exosomes isolated from each group (from $4 \mathrm{ml}$ serum) were then re-suspended in $1 \mathrm{ml} 1 \mathrm{X}$ PBS. Subsequently, $20 \mu \mathrm{l}$ sample was dropped onto a copper grid and left to subside for $\sim 1 \mathrm{~min}$ at room temperature. Filter paper was used to absorb the remaining fluid, followed by fixation in $2.5 \%$ glutaraldehyde Sorensen's phosphate buffer for $1 \mathrm{~h}$ at $4^{\circ} \mathrm{C}$. Samples were dehydrated with different concentrations (70-100\%) of acetone and embedded in Epon 812 at $60^{\circ} \mathrm{C}$ for $24 \mathrm{~h}$. Sections of $60-\mathrm{nm}$ thickness were prepared using an ultra-microtome. Then, the sections were lightly counter-stained with uranyl acetate (for $25 \mathrm{~min}$ ) and lead citrate (for $5 \mathrm{~min}$ ) at room temperature and were observed using a FEI Tecnai G2 Spirit Bio-Twin transmission electron microscope (Thermo Fisher Scientific, Inc.).

Western blotting. Western blotting was performed as previously described (28). Briefly, serum exosomes or cells were lysed with RIPA protein extraction buffer (Beijing Solarbio Science \& Technology Co., Ltd.) and incubated for $30 \mathrm{~min}$ at $4^{\circ} \mathrm{C}$. Cell debris was removed by centrifugation at $4^{\circ} \mathrm{C}$ for $8 \mathrm{~min}$ at $13,400 \mathrm{x}$ g. Protein concentrations of the supernatant were determined using a BCA protein assay reagent kit (Beijing Solarbio Science \& Technology Co., Ltd.) according to the manufacturer's protocol. Proteins (30 $\mu \mathrm{g}$ per lane) were separated via $10 \%$ SDS-PAGE and transferred to PVDF membranes $(0.45 \mu \mathrm{m})$. After blocking with $5 \%$ skimmed milk in TBST $(0.1 \%$ Tween-20) at room temperature for $1 \mathrm{~h}$, the membranes were incubated at $4^{\circ} \mathrm{C}$ overnight with primary antibodies targeted against: TSG101 (1:1,000), CD63 (1:1,000), CD9 (1:2,000), Collagen I (1:200), Collagen III (1:800), a-SMA (1:200), PIK3CA (1:400), Akt (1:1,000), p-Akt (ser 473, 1:1,000), p-Akt (thr308, 1:1,000), mTOR (1:1,000), p-mTOR (ser 2481, 1:1,000), p-mTOR (ser 2448, 1:1,000), GAPDH $(1: 1,000)$ and $\beta$-actin $(1: 1,000)$. Following washing three times with TBST, the membranes were incubated with a secondary antibody for $1 \mathrm{~h}$ at room temperature, then washed three more times with TBST. Protein bands were visualized using enhanced chemiluminescence detection agents (EMD Millipore). Protein expression was semi-quantified using ImageJ version 1.5.2 software (National Institutes of Health) with $\beta$-actin or GAPDH as the loading control.

Bioinformatics and statistics. National Center for Biotechnology Information Gene Expression Omnibus (GEO) datasets (29) and the Human MicroRNA Disease Database (HMDD) version 3.2 (30) were searched using 'Non-coding RNA profiling by array and CHF' as the key word. The results were individually analyzed using the GEO2R analytical tool, which is a R-based interactive web tool that identifies differentially expressed miRNAs under various experimental conditions. Subsequently, sample groups were assigned to $\mathrm{CHF}$ and control groups, and the top 195 differentially expressed miRNAs in HMDD were selected. Moreover, the datasets of GEO were deal with R version 3.4.4 for Windows. Altered gene expression levels displaying significant changes $(\mathrm{P}<0.05$ and $\mid \mathrm{LogFCl}>2)$ were considered as differentially expressed. volcano plot was generated using the ggplot 2 packages in R-3.4.4. The 30 most markedly differentially expressed miRNAs from GEO dataset were screened and the heat map was generated using gplots and RColorBrewer packages in R-3.4.4. Subsequently, the datasets of miRNAs collected from HMDD and the top 30 miRNAs from GEO were assessed using a Venn diagram package in R-3.4.4.

An advanced search for the association between these miRNAs and CHF was performed, and miR-320a was selected for subsequent experiments. The target genes of miR-320a were predicted using Target Scan version 7.2 (31), miRwalk (32) and microRNA Data Integration Portal (miRDIP) databases (33) with different algorithms. In order to decrease false-positive results, only genes that appeared in all three databases were used for subsequent analysis. The target genes were then analyzed using Database for Annotation, Visualization and Integrated Discovery (DAVID; version 6.8) (34) and Kyoto Encyclopedia of Genes and Genomes (KEGG) (35). Pathways were selected as functional annotation categories for this analysis. Only pathways that displayed significant differences $(\mathrm{P}<0.05)$ were functionally annotated. A binding site of miRNA-320a was identified in the 3'-untranslated region of PIK3CA was predicted using TargetScan version 7.2 (31).

Cell culture. The human fetal myocardial fibroblast cell line (HEH2) was purchased from Shanghai Tongwei Biotechnology Co., Ltd. Cells were cultured in DMEM supplemented with $1 \%$ penicillin-streptomycin and $10 \% \mathrm{FBS}$ at $37^{\circ} \mathrm{C}$ with $5 \% \mathrm{CO}_{2}$.

Exosome labeling and transfer to HEHE2 cells. Exosomes from $\mathrm{CHF}$ and healthy patient groups were labeled with PKH67 green fluorescent membrane linker-dye (Sigma-Aldrich; Merck KGaA) according to the manufacturer's protocol. Purified exosomes were labeled with PKH67 dye by adding $500 \mu \mathrm{l}$ Diluent $\mathrm{C}$ and $4 \mu \mathrm{l}$ diluted PKH67 dye and incubated for $4 \mathrm{~min}$ at room temperature. Labeled purified exosomes were washed in PBS, pelleted at $100,000 \times \mathrm{g}$ for $60 \mathrm{~min}$ at $4^{\circ} \mathrm{C}$ and resuspended in PBS to a final volume of $200 \mu \mathrm{l}$. The protein concentration of exosomes was quantified using a BCA 
protein assay reagent kit (Beyotime Institute of Biotechnology) according to the manufacturer's protocol. PKH67-stained exosomes $(40 \mu \mathrm{g})$ were incubated for $1 \mathrm{~h}$ or $24 \mathrm{~h}$ with $\mathrm{HEH} 2$ cells at $37^{\circ} \mathrm{C}$ (except the control group) and then washed twice with PBS. All cells were then fixed with $4 \%$ formaldehyde for $20 \mathrm{~min}$ at room temperature. The nuclei were stained with DAPI (Beijing Solarbio Science \& Technology Co., Ltd.). Fluorescence was visualized using an IX53 confocal microscope (Olympus Corporation).

Cell viability assay. HEH2 cell viability was assessed by

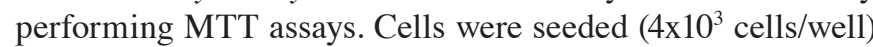
into 96 -well plates and cultured for $24 \mathrm{~h}$. Following transfection, $20 \mu \mathrm{l}$ MTT solution was added into each well and incubated for $4 \mathrm{~h}$ at $37^{\circ} \mathrm{C}$. Subsequently, the culture medium was carefully aspirated and $150 \mu 1 \mathrm{DMSO}$ was added to each well to dissolve the purple formazan. The absorbance of each well was measured at a wavelength of $490 \mathrm{~nm}$ using a microplate reader. Cell viability in the control group was set as $100 \%$.

Quantification of apoptosis. Apoptotic cells were quantified by analyzing PI staining via flow cytometry. Following transfection, cells were collected, washed with PBS and resuspended in $100 \mu \mathrm{l}$ binding buffer. Subsequently, cells were double-stained with Annexin V and PI using the Annexin V-PI apoptosis detection kit (Vazyme Biotech Co., Ltd.) according to the manufacturer's protocol. Apoptotic cells were analyzed using a flow cytometer (Beckman Coulter FC500 Cytometer; Beckman Coulter, Inc.). Annexin $\mathrm{V}^{+} / \mathrm{PI}^{-}$staining indicated that cells were undergoing apoptosis (early stage of apoptosis), Annexin $\mathrm{V}^{+} / \mathrm{PI}^{+}$staining indicated that cells were undergoing late stage of apoptosis, necrosis or had already died, and Annexin $\mathrm{V}^{-} / \mathrm{PI}^{-}$staining indicated that cells were alive and not undergoing measurable apoptosis. Results were processed using CXP analysis software (version 2.1) from Beckman Coulter. For each analysis, 20,000 events were collected.

$R N A$ extraction and reverse transcription-quantitative PCR $(R T-q P C R)$. Total RNA was extracted from serum samples or HEH2 cells using an miRcute miRNA extraction kit (Tiangen Biotech Co., Ltd., cat. no. DP501). Total RNA was reverse transcribed into cDNA using the miRcute miRNA First-Strand cDNA Synthesis kit (Tiangen Biotech Co., Ltd, cat. no. KR201) according to the manufacturer's protocol. Subsequently, qPCR was performed using miRcute miRNA qPCR Detection kit (SYBR-Green, Tiangen Biotech Co., Ltd, cat. no. FP401) and the ABI7500 Real Time PCR System (both Applied Biosystems; Thermo Fisher Scientific, Inc.). Cycling protocols were $2 \mathrm{~min}$ at $94^{\circ} \mathrm{C}$ followed by 40 cycles of $20 \mathrm{sec}$ at $94^{\circ} \mathrm{C}, 34 \mathrm{sec}$ at $60^{\circ} \mathrm{C}$ and a final extension of $7 \mathrm{~min}$ at $72^{\circ} \mathrm{C}$. miRNA and mRNA expression levels were quantified using the $2^{-\Delta \Delta \mathrm{Cq}}$ method and normalized to the internal reference genes U6 and GAPDH, respectively (36).

miR-320a mimics and inhibitor transfection. HEH2 cells $\left(5 \times 10^{4}\right.$ cells/well) were seeded into 24 wells for $24 \mathrm{~h}$ and transfected with miR-320a inhibitor (40 nM; 5'-UCGCCC UCUCAACCCAGCUUUU-3'), miR-320a mimics (40 nM; forward, 5'-AAA AGC UGGGUUGAGAGGGCGA-3' and reverse, 5'-GCCCUCUCAACCCAGCUUUUUU-3'), NC-mimics (40 nM; forward, 5'-UUCUCCGAACGUGUC ACGUTT-3' and reverse, 5'-ACGUGACACGUUCGGAGA ATT-3') or NC-inhibitor (40 nM; 5'-CAGUACUUUUGU GUAGUACAA-3') for $6 \mathrm{~h}$ at $37^{\circ} \mathrm{C}$ using Lipofectamine 2000 transfection reagent (Invitrogen; Thermo Fisher Scientific, Inc.) according to the manufacturer's protocol. miR-320a mimics were double-stranded RNA oligos, whereas miR-320a inhibitors were single-stranded. The inhibitor was modified by 2'-O-methylation. At $72 \mathrm{~h}$ post-transfection, miR-320a expression levels were assessed via RT-qPCR.

ELISA. The content of soluble growth stimulation expressed gene 2 (sST2) in serum samples and HEH2 cells was determined by performing ELISAs. Briefly, cells were seeded $\left(2 \times 10^{5}\right.$ cells/well) into 6 -well plates and allowed to adhere overnight. Following transfection, $500 \mu \mathrm{l}$ PBS was added to $1 \times 10^{6}$ cells, followed by lysis by ultrasonication. Cell debris was removed via centrifugation at $4^{\circ} \mathrm{C}$ for $10 \mathrm{~min}$ at $10,000 \mathrm{x} \mathrm{g}$. The content of sST2 in the supernatants of $\mathrm{HEH} 2$ cells and serum samples obtained from CHF and healthy control groups were detected by performing ELISAs according to the manufacturer's instructions.

Statistical analysis. Statistical analyses were performed using Microsoft Excel (Microsoft Corporation) and GraphPad Prism software (version 5; GraphPad Software, Inc.). Data are presented as the mean \pm SEM. All experiments were performed in triplicate. Comparisons between two groups were analyzed using the unpaired Student's t-test. Comparisons among multiple groups were analyzed using one-way ANOVA followed by Tukey's post hoc test. $\mathrm{P}<0.05$ was considered to indicate a statistically significant difference.

\section{Results}

Bioinformatics prediction of miRNA. The gene expression profile of GSE104150, 'Association of miR-197-5p, a circulating biomarker for heart failure, with myocardial fibrosis and adverse cardiovascular events among patients with stage C or D heart failure', was obtained from GEO datasets. The GSE104150 dataset contained blood from humans and was comprised of 16 samples, including 7 healthy control tissues and 9 CHF tissues. After the GSE104150 dataset was analyzed, 185 genes were selected for inclusion in a volcano plot (Fig. 1A). A heatmap displaying the top 30 differentially expressed genes was generated (Fig. 1B). A differential expression miRNA analysis was performed using GEO and HMDD, resulting in the identification of 195 and 30 miRNAs, respectively. Among the identified miRNAs, both GEO and HMDD identified these 2 miRNAs as being differentially expressed (Fig. 1C and D). As hsa-miR-320a are reported to have closely related to cardiac diseases $(24,25)$, miR-320a was selected for further investigation.

Correlation analysis of the expression levels of serum miR-320a and clinical characteristics in patients with $C H F$. To confirm the prediction in the present study, the serum expression levels of miR-320a in healthy volunteers and patients with CHF were detected. The expression of miR-320a was significantly elevated in patients with $\mathrm{CHF}$ compared 
A

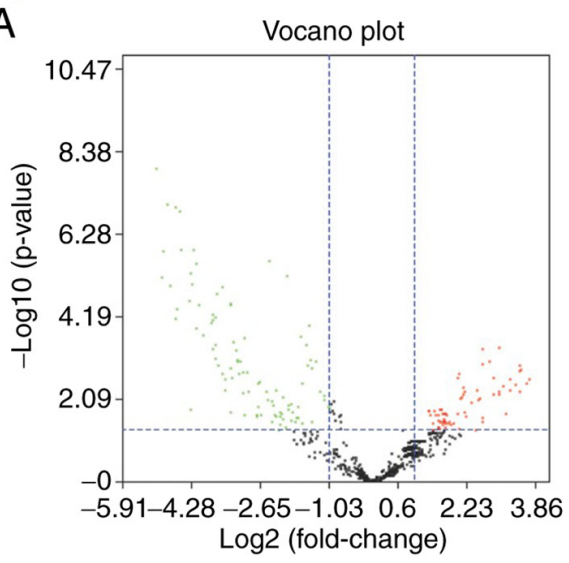

C

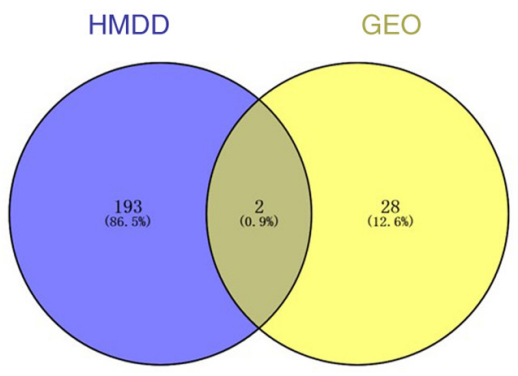

$\mathrm{D}$
$\mathrm{B}$

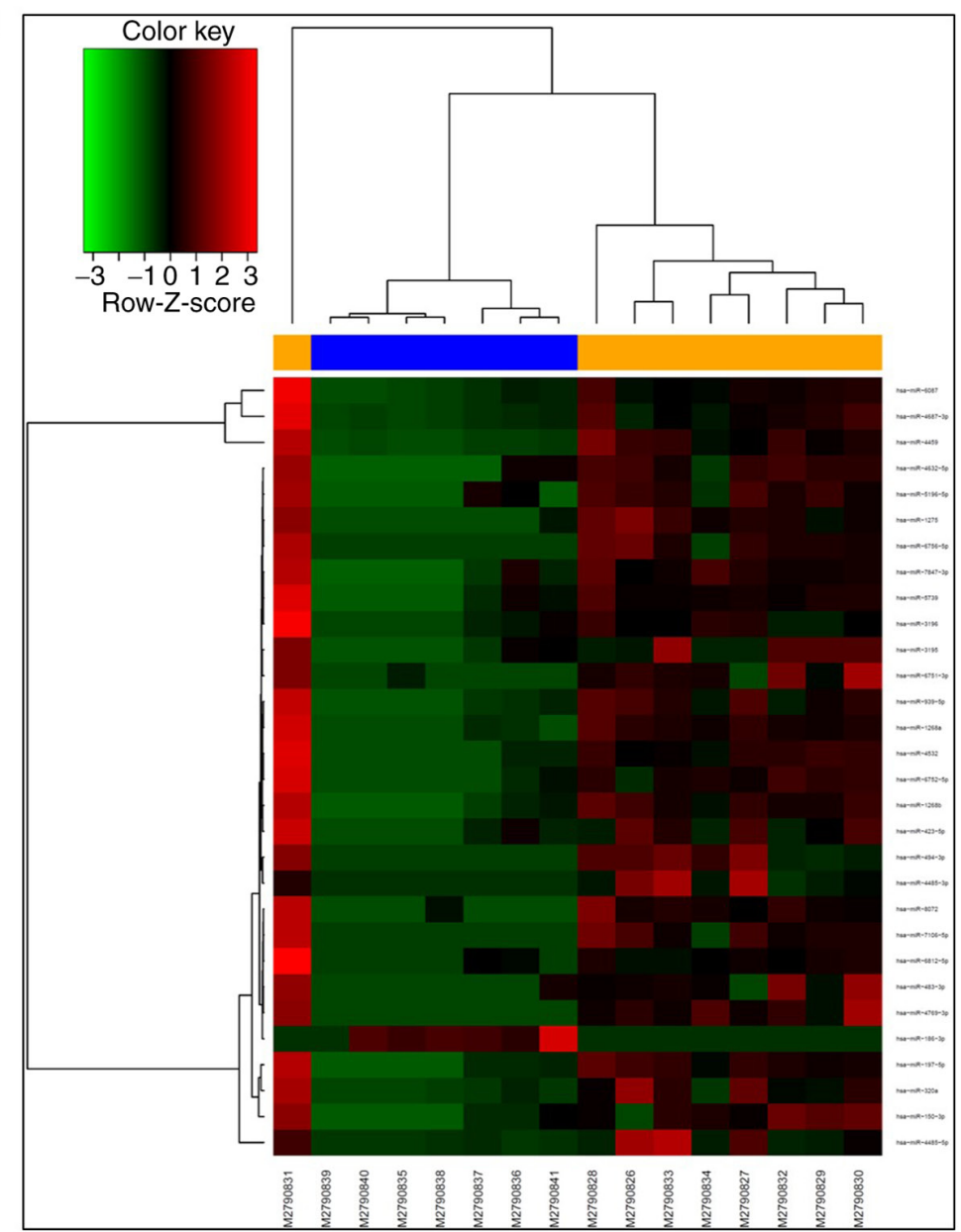

Figure 1. Bioinformatics prediction of miRNA. (A) Volcano plot displaying the differential expression of miRNAs in CHF and healthy control groups using the ggplot2 package in R. The $\mathrm{x}$ axis presents the mean fold-change in miRNA expression between CHF and healthy control groups [log2 (Fold Change) and $\mathrm{P}<0.05]$. (B) Heat map of the expression levels of the top 30 differentially expressed miRNAs drawn using gplots and RColorBrewer packages. Red, green and black indicate upregulation, downregulation and no change, respectively (C) Venn diagram of the differentially expressed miRNAs in GEO and HMDD. (D) Differentially expressed miRNAs identified by GEO and HMDD. miRNA/miR, microRNA; CHF, chronic heart failure; GEO, Gene Expression Omnibus; HMDD, Human MicroRNA Disease Database.

with healthy controls $(\mathrm{P}<0.05$; Fig. 2A). Furthermore, the present study also assessed the serum levels of sST2, which is a biomarker of cardiac remodeling and fibrosis (37). The results demonstrated that the serum levels of sST2 were also significantly increased in patients with CHF compared with healthy controls $(\mathrm{P}<0.01$; Fig. 2B).

LVEF, LVEDD, NT-proBNP, sST2 and NYHA classification are diagnostic markers for CHF (38-41). The association between serum miR-320a expression levels and the clinical characteristics of patients with CHF was analyzed in the present study. The expression of serum miR-320a was significantly correlated with the clinical characteristics of patients with CHF. Serum miR-320a expression levels were positively correlated with LVEDD ( $\mathrm{r}=0.841$; $\mathrm{P}<0.01$; Fig. 2C), sST2 ( $\mathrm{r}=0.688$; $\mathrm{P}<0.05$; Fig. 2D), NT-proBNP ( $\mathrm{r}=0.848$; $\mathrm{P}<0.01$; Fig. $2 \mathrm{~F}$ ) and NYHA classification $(\mathrm{r}=0.827 ; \mathrm{P}<0.01 ;$ Fig. $2 \mathrm{E})$, but negatively correlated with LVEF ( $\mathrm{r}=-0.760 ; \mathrm{P}<0.05$; Fig. 2G).

Exosome sample identification and the expression levels of miR-320a in exosomes and exosome-depleted supernatants. Subsequently, the characteristics of exosomes were determined by performing particle-size analysis, transmission electron microscopy and western blotting. Exosomes in the serum displayed a double layer membrane and ranged from 40-200 $\mathrm{nm}$ in size (Fig. 3A and C). CD63, CD9 and TSG101, which are protein markers of exosomes (42), were used to evaluate exosomes via western blotting (Fig. 3B). The results revealed that CD63, CD9 and TSG101 were positively expressed in the exosomes of the healthy control and $\mathrm{CHF}$ groups. Collectively, these results suggested that exosomes were successfully extracted from the serum. To characterize the primary expression pattern of miR-320a in serum, its expression levels in exosomes and exosome-depleted supernatants (residues) were determined. The expression level of miR-320a was significantly increased in normal (NOR) and CHF exosomes compared with NOR and CHF exosome-depleted supernatants (residues), respectively, suggesting that miR-320a was primarily expressed in the exosomes (Fig. 3D).

Transfection of exosomes into cardiac fibroblasts and the expression of miR-320a in HEH2 cells. PKH lipophilic dyes, which are highly fluorescent and stain exosomes membranes by intercalating their aliphatic portion into the exposed lipid 
A

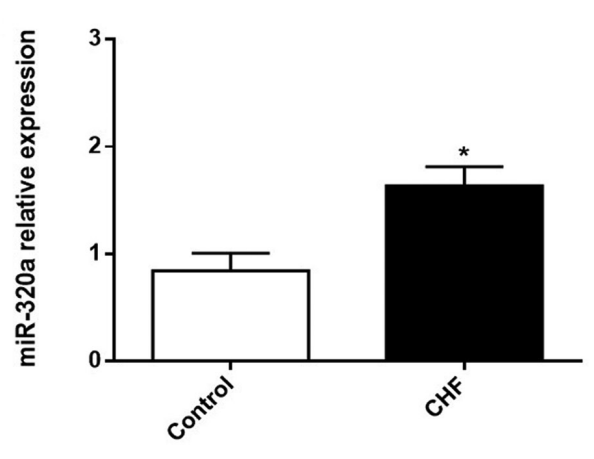

C

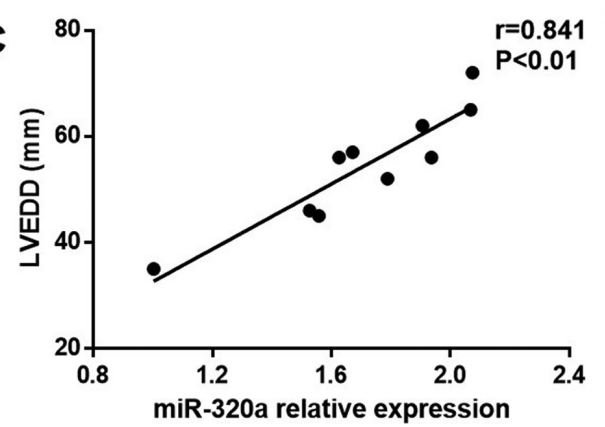

E
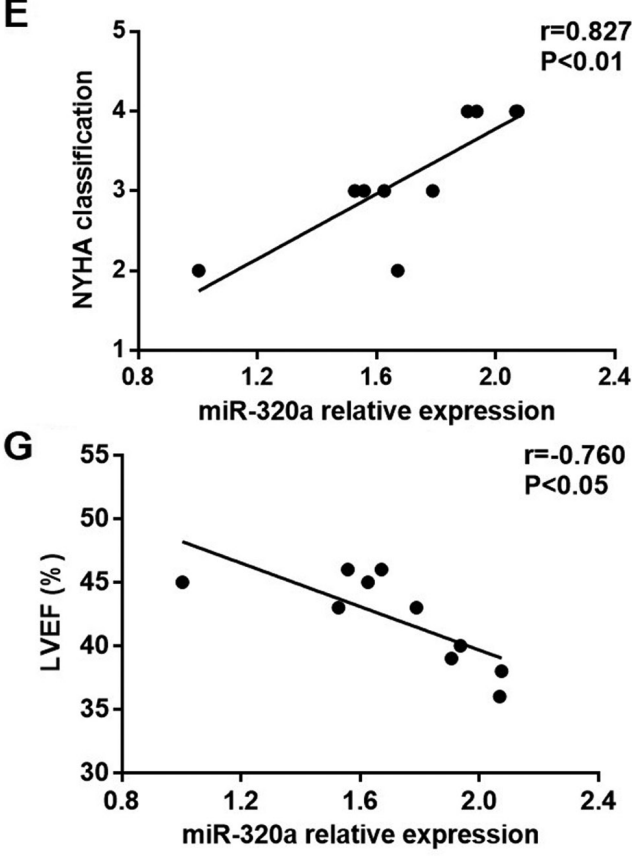

B
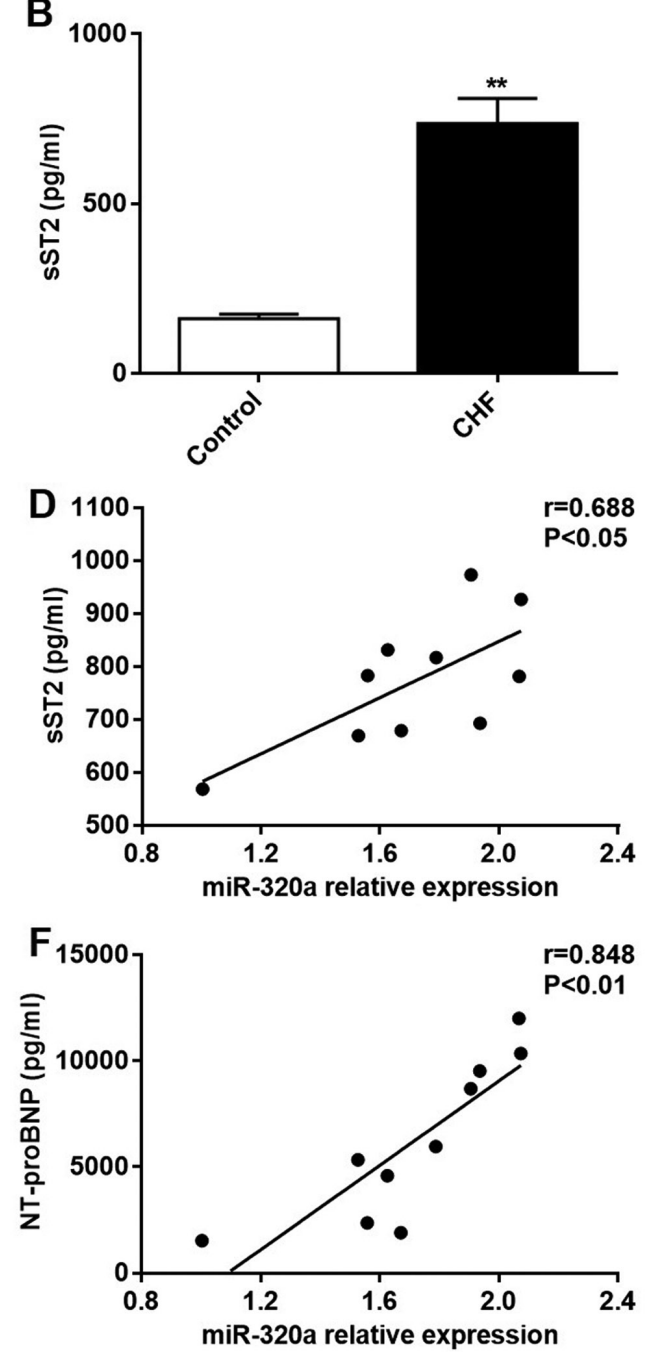

Figure 2. Correlation analysis of the expression levels of serum miR-320a and clinical characteristics in patients with CHF. miR-320a expression levels and the content of sST2 in serum samples collected from patients with CHF and healthy controls were detected via reverse transcription-quantitative PCR and ELISA, respectively. LVEF and LVEDD parameters were measured using Color Doppler Ultrasonic Diagnostic Equipment. New York Heart Association classification of patients with CHF was based on their limitations during physical activity according to the 2017 Chinese CHF Diagnosis and Treatment guidelines. Serum levels of NT-proBNP were determined by performing an electrochemiluminescence immunoassay. (A) miR-320a expression levels and (B) the content of sST2 in serum samples collected from patients with CHF and healthy controls. Correlation between miR-320a and (C) LVEDD, (D) sST2, (E) NYHA classification, (F) NT-proBNP or (G) LVEF. Data are presented as the mean \pm SEM from at least three independent experiments. Comparisons between two groups were analysed using one-way ANOVA followed by Tukey's post hoc test. Pearson's correlation coefficient was used to assess correlations. "P<0.05 and ${ }^{* *} \mathrm{P}<0.01$ vs. control. miR, microRNA; CHF, chronic heart failure; sST2, soluble growth stimulation expressed gene 2; LVEDD, left ventricular end-diastolic diameter; NYHA, New York Heart Association; NT-proBNP, NT-proB-type natriuretic peptide; LVEF, left ventricular ejection fraction.

bilayer (43), were employed to evaluate the ability of exosomes to transfer miR-320a into cardiac fibroblasts. The results revealed that the NOR and CHF exosomes were successfully absorbed by HEH2 cells in a time-dependent manner (Fig. 4A). Subsequently, the present study detected the expression levels of miR-320a in HEH2 cells following incubation with serum exosomes from NOR and CHF groups for $12 \mathrm{~h}$. Compared with the control group, the expression levels of miR-320a in NOR and CHF exosomes were significantly upregulated (both $\mathrm{P}<0.01$ ), and the expression levels of miR-320a in the CHF exosomes group were significantly higher compared with the NOR exosomes group $(\mathrm{P}<0.01)$ (Fig. 4B). 

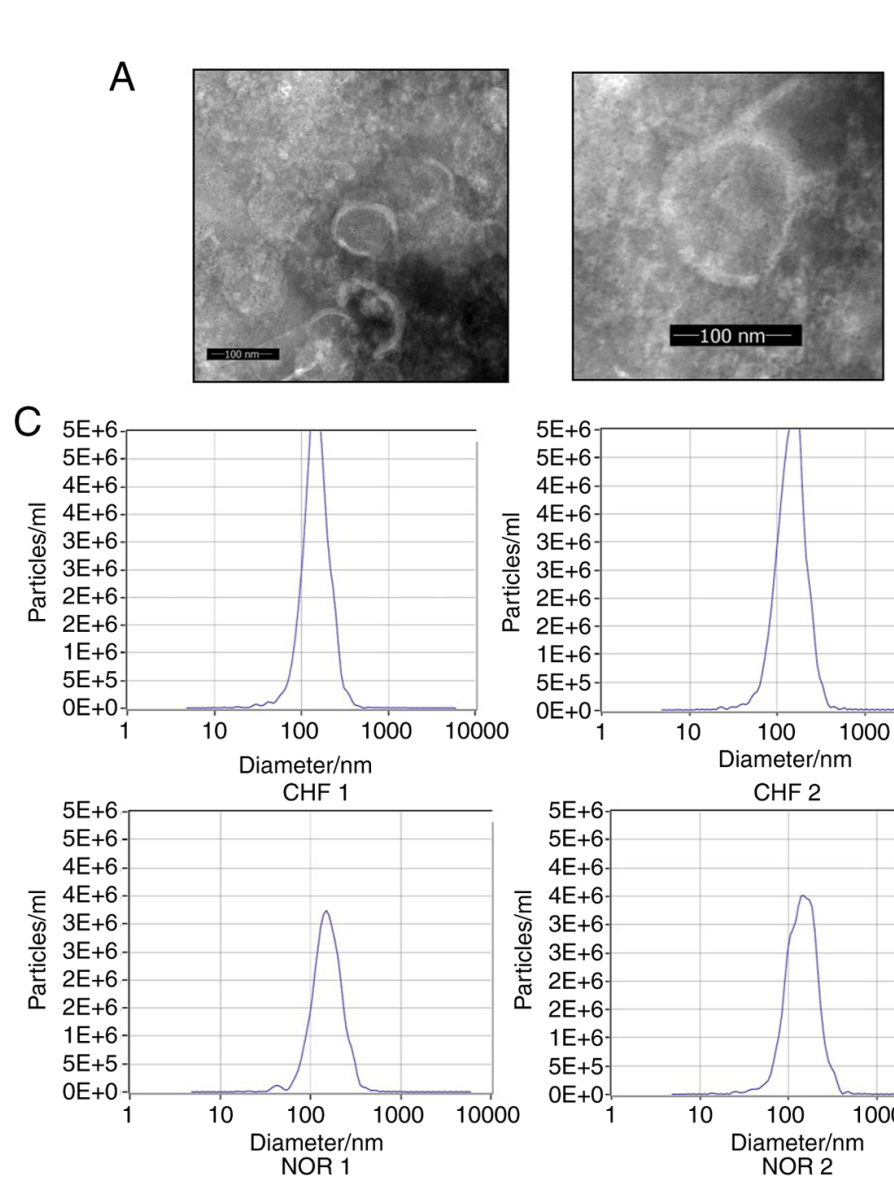

B

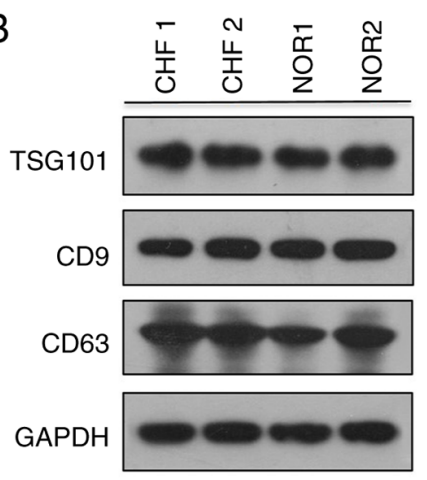

Figure 3. Characterization of exosomes extracted from serum samples, and the expression levels of miR-320a in exosomes and exosome-depleted supernatant. Exosomes were extracted from serum samples of patients with CHF and normal controls using the exoRNeasy Serum-Plasma Midi kit. (A) Exosomes from NOR control group were observed via transmission electron microscopy. (B) Exosome biomarkers, including CD63, CD9 and TSG101, were identified via western blotting in CHF and normal groups (two patients each group, CHF1-2 and Normal 1-2, respectively). (C) Particle sizes of extracted exosomes from CHF and normal groups (two patients each group) were confirmed via Nanoparticle Size Analyzer. (D) Relative expression levels of miR-320a in exosomes and exosome-depleted supernatants (residues) were determined via reverse transcription-quantitative PCR. Data are presented as the mean \pm SEM from at least three independent experiments. Comparisons among multiple groups were analysed using one-way ANOVA followed by Tukey's post hoc test. ${ }^{\# \#} \mathrm{P}<0.01$ vs. Normal exosomes; ${ }^{\dagger} \mathrm{P}<0.01$ vs. CHF exosomes. miR, microRNA; CHF, chronic heart failure; TSG101, tumor susceptibility 101.

Effects of miR-320a on cell viability, intracellular sST2 content and apoptosis in HEH2 cells. To determine the effect of miR-320a on cardiac fibroblast proliferation, $\mathrm{HEH} 2$ cells were transfected with miR-320a mimics or inhibitor. Compared with the NC-inhibitor group, cell viability was significantly decreased following transfection with miR-320a inhibitor for $72 \mathrm{~h}(\mathrm{P}<0.05$; Fig. 5A). By contrast, compared with the NC-mimics group, miR-320a overexpression did not significantly alter HEH2 cell viability $(\mathrm{P}>0.05)$. Following transfection with miR-320a mimics or inhibitor, the percentage of apoptotic myocardial fibroblasts was significantly increased compared with the NC-mimics and NC-inhibitor group, respectively (both $\mathrm{P}<0.01$; Fig. 5C and D). Notably, compared with miR-320a mimics-transfected cells, miR-320a knockdown significantly increased the percentage of apoptotic cells $(\mathrm{P}<0.01)$. In addition, the sST2 content was significantly elevated following transfection with miR-320a mimics compared with the NC-mimics group $(\mathrm{P}<0.01$; Fig. 5B). Following transfection with miR-320a inhibitor, the sST2 content was significantly decreased in $\mathrm{HEH} 2$ cells compared with the NC-inhibitor group $(\mathrm{P}<0.01)$.
Effect of miR-320a on target gene (PIK3CA) expression in $\mathrm{HEH} 2$ cells. To investigate the potential mechanisms underlying miR-320a-induced myocardial fibroblast proliferation, bioinformatics tools (TargetScan, miRwalk and miRDIP) were used to predict the target genes of miR-320a. The results identified 592 possible target genes of miR-320a (Fig. 6A). Enriched pathways and processes significantly associated with each predicted target gene were identified by performing DAVID and KEGG analyses. Several common signaling pathways were identified, including the 'PI3K/Akt signaling pathway', 'insulin signaling pathway' and 'endocytosis' (Fig. 6B). Among these pathways, PI3K/Akt, which has been demonstrated to be closely associated with myocardial fibrosis (44), was further investigated. Predicted genes of PI3K/Akt pathway were shown in Fig. 6D. PIK3CA, an integral part of the PI3K/Akt pathway (45), was selected for subsequent investigation.

A predicted binding site of miRNA-320a was identified in the 3'-untranslated region of PIK3CA (Fig. 6C). Furthermore, the effects of miR-320a mimics and inhibitor on miR-320a expression were confirmed via RT-qPCR. miR-320a mimics significantly increased the expression of miR-320a in HEH2 
A
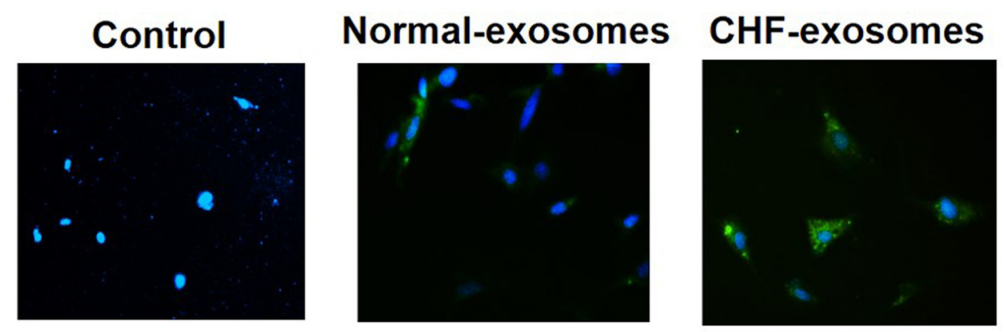

$12 \mathrm{~h}$
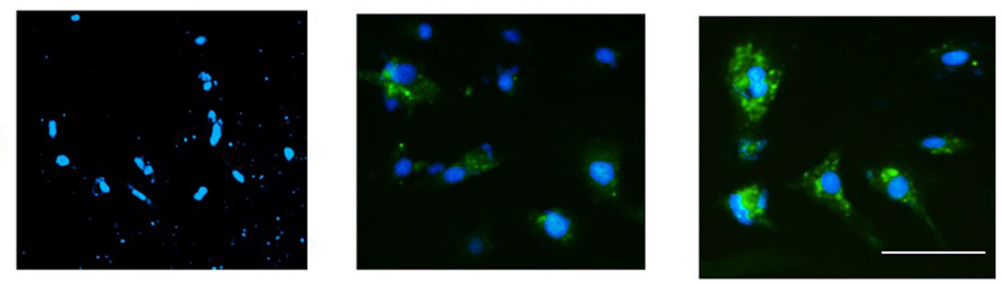

B

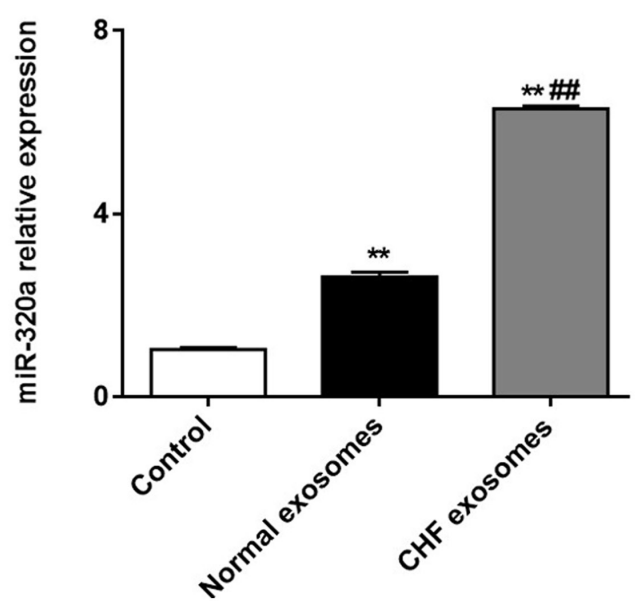

Figure 4. Transfection of exosomes into cardiac fibroblasts and the expression of miR-320a in HEH2 cells. (A) Following incubation with PKH67-stained exosomes for $12 \mathrm{~h}$, cells were visualized via fluorescence microscopy. Scale bar, $100 \mu \mathrm{m}$. (B) Following incubation with NOR or CHF exosomes for $12 \mathrm{~h}$, the expression levels of miR-320a were quantified via reverse transcription-quantitative PCR. Data are presented as the mean \pm SEM from at least three independent experiments. Comparisons among multiple groups were analysed using one-way ANOVA followed by Tukey's post hoc test. ${ }^{* * *} \mathrm{P}<0.01 \mathrm{vs}$. control; ${ }^{\# \#} \mathrm{P}<0.01$ vs. normal exosomes; miR, microRNA.

cells compared with the NC-mimics group $(\mathrm{P}<0.01)$, whereas miR-320a inhibitor significantly decreased miR-320a expression compared with the NC-inhibitor group $(\mathrm{P}<0.01)$ (Fig. 7A). Furthermore, the effect of miR-320a mimics or inhibitor transfection on PIK3CA expression in $\mathrm{HEH} 2$ cells was detected to verify PIK3CA as the direct target gene of miR-320a. miR-320a overexpression significantly upregulated the expression of PIK3CA compared with the NC-mimics group $(\mathrm{P}<0.01)$, whereas miR-320a knockdown significantly downregulated the expression of PIK3CA in $\mathrm{HEH} 2$ cells compared with the NC-inhibitor group $(\mathrm{P}<0.01)$ (Fig. 7B). In addition, the correlation between miR-320a and PIK3CA was analyzed. The results revealed that there was a significant positive correlation between miR-320a and PIK3CA expression ( $r=0.923$; $\mathrm{P}<0.01$; Fig. 7C).

miR-320a promotes myocardial fibroblast proliferation via regulating the PIK3CA/Akt/mTOR signaling pathway. To further investigate the mechanism underlying miR-320a in myocardial fibroblast proliferation, the involvement of the PIK3CA/Akt/mTOR signaling pathway was investigated. Cells transfected with miR-320a mimics displayed significantly increased PIK3CA protein expression levels compared with the
NC-mimics group ( $\mathrm{P}<0.01 ;$ Fig. 8). The expression of PIK3CA was significantly decreased in miR-320a inhibitor-transfected $\mathrm{HEH} 2$ cells compared with NC-inhibitor-transfected HEH2 cells $(\mathrm{P}<0.01)$. miR-320a overexpression significantly increased the ratios of $\mathrm{p}$-Akt (ser 473)/Akt and p-Akt (ser 308)/Akt compared with the NC-mimics group (both $\mathrm{P}<0.01)$. By contrast, miR-320a knockdown significantly decreased the ratios of $\mathrm{p}-\mathrm{Akt}(\mathrm{ser} 473) / \mathrm{Akt}(\mathrm{P}<0.01)$ compared with the NC-inhibitor group. When compared with the NC-inhibitor group, the ratio of p-Akt (ser 308)/Akt was also reduced after miR-320a inhibitor transfection, although the difference was not significant $(\mathrm{P}>0.05)$. Furthermore, the ratio of p-mTOR (ser 2448)/mTOR was significantly elevated following miR-320a mimics transfection compared with the NC-mimics group $(\mathrm{P}<0.01)$, but notably downregulated following miR-320a inhibitor transfection, although this was not significant compared with the NC-inhibitor group ( $\mathrm{P}>0.05$ ). Moreover, the ratio of p-mTOR (ser 2481)/mTOR was not changed after miR-320a mimic and inhibitor transfection ( $P>0.05)$, suggesting that p-mTOR (ser 2481) may not be the target protein of miR-320a. The western blotting results revealed that the expression levels of the downstream 
A

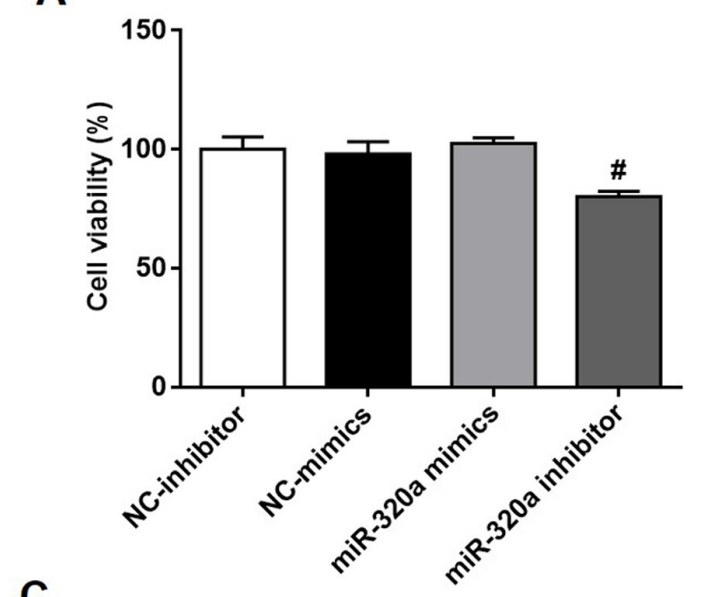

B

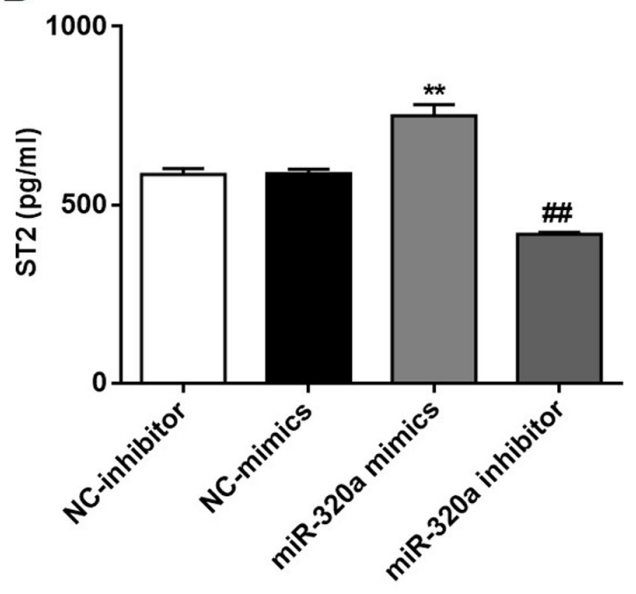

C
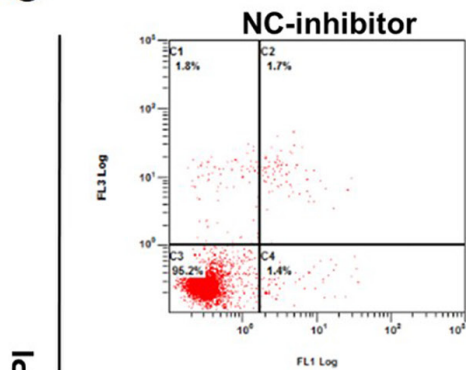

miR-320a mimics

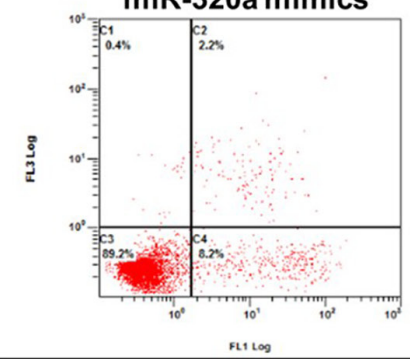

NC-mimics

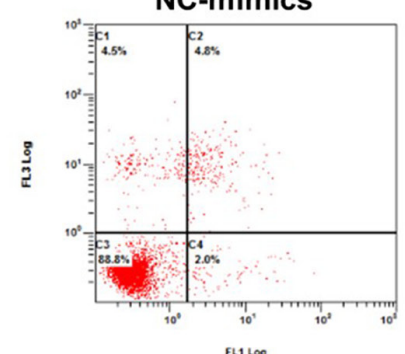

miR-320a inhibitor

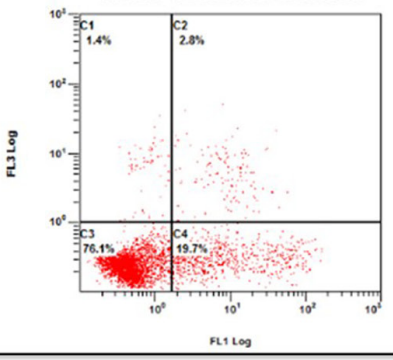

D

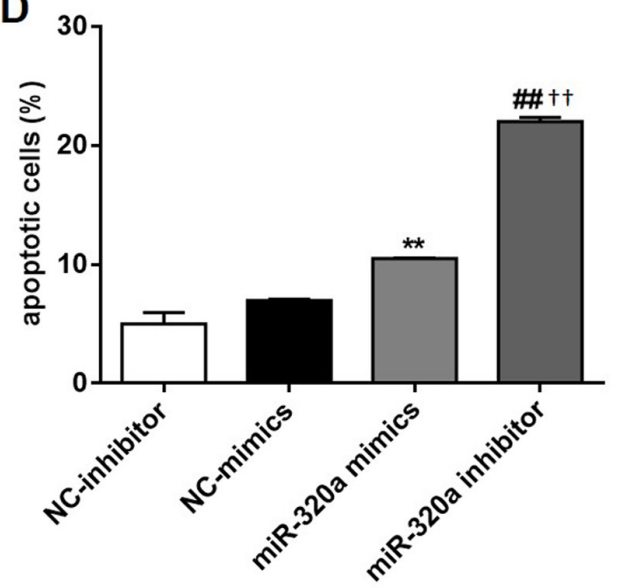

AnnexinV-FITC

Figure 5. miR-320a knockdown decreases cell viability and intracellular sST2 content, as well as enhances apoptotic cell death in HEH2 cells. HEH2 cells were transfected with NC-mimics, NC-inhibitor, miR-320a mimics or miR-320a inhibitor for $72 \mathrm{~h}$. (A) Cell viability was determined by performing the MTT assay. (B) sST2 content in HEH2 cells was assessed by performing ELISAs. Cell apoptosis (C) was determined via flow cytometry and quantified. (D) The apoptosis rate of cells including early and late stage of apoptotic cells. Data are presented as the mean \pm SEM from at least three independent experiments. Comparisons between two groups were analysed using the unpaired Student's t-test. ${ }^{* *} \mathrm{P}<0.01$ vs. NC-mimics; ${ }^{\#} \mathrm{P}<0.05$ and ${ }^{\# \#} \mathrm{P}<0.01$ vs. NC-inhibitor; ${ }^{\dagger \dagger} \mathrm{P}<0.01$ vs. miR-320a mimics. miR, microRNA; sST2, soluble growth stimulation expressed gene 2; NC, negative control.

proteins of mTOR, including collagen I and collagen III, were significantly increased following transfection with miR-320a mimics compared with NC-mimics (both $\mathrm{P}<0.01$ ), whereas transfection with miR-320a inhibitor significantly decreased the expression levels of collagen I and collagen III in HEH2 cells compared with the NC-inhibitor group (both $\mathrm{P}<0.01$. In addition, compared with the NC-mimics and NC-inhibitor groups, the expression of $\alpha$-SMA was significantly increased $(\mathrm{P}<0.01)$ and decreased $(\mathrm{P}<0.01)$ following transfection with miR-320a mimics and miR-320a inhibitor, respectively.

\section{Discussion}

The miR-320 family is conserved, but only exists in vertebrates, from Xenopus to humans (46). This miRNA family consists of five members, and miR-320a regulated the abundant biological processes in cardiovascular diseases, including atherogenesis (47), drug-induced cardiotoxicity (48) and arrhythmia (49). The present study investigated the effect of miR-320a on myocardial fibroblast proliferation in HEH2 cells, as well as the underlying molecular mechanisms.

Due to their stability in human plasma and serum, miRNAs are potential biomarkers for a number of diseases, such as $\mathrm{CHF}$ and autism spectrum disorder $(24,50)$. In the present study, the results obtained from the bioinformatics prediction revealed that miR-320a may be differentially expressed between patients with $\mathrm{CHF}$ and healthy controls. To improve the reliability of the bioinformatics prediction, the expression of serum miR-320a in the CHF and healthy control groups was detected. The results demonstrated that the expression of serum miR-320a in patients with CHF was significantly elevated compared with the healthy control group, which confirmed the bioinformatics prediction. To further investigate 
A

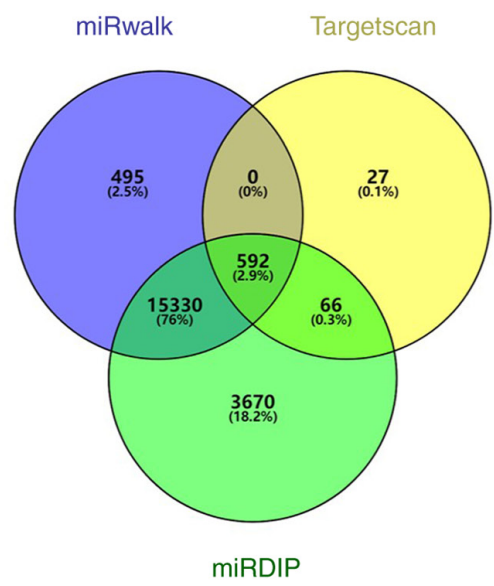

C

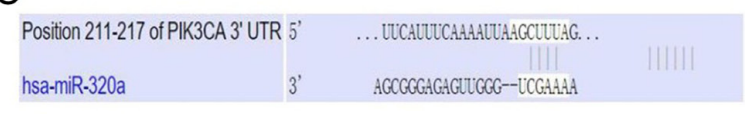

B

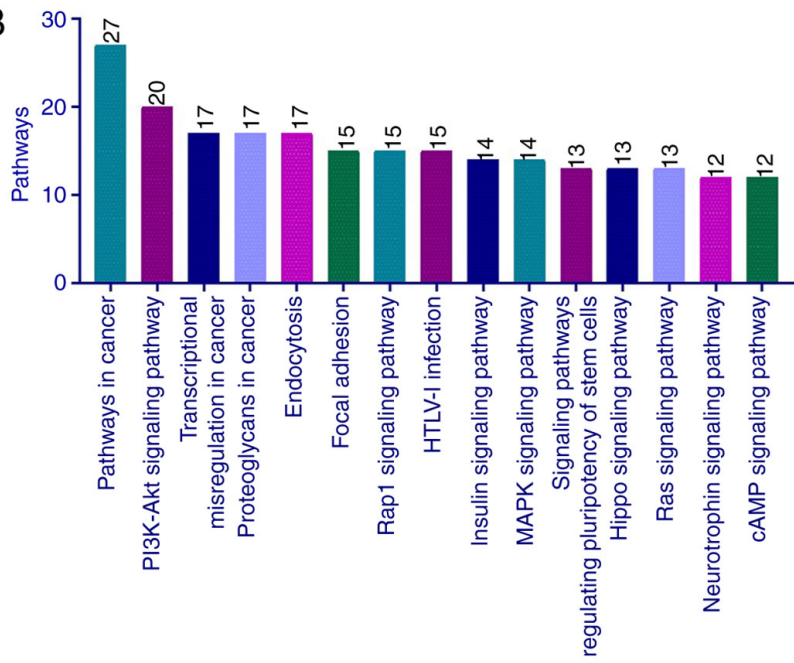

\begin{tabular}{llll}
\hline & \multicolumn{3}{l}{ Prediction genes of PI3K/Akt pathway } \\
\hline YWHAZ & RXRA & KITLG & CDK6 \\
IL6R & ITGB3 & YWHAE & PTEN \\
EIF4B & MAPF1 & YWHAH & CASP9 \\
TSC1 & CCND2 & PPP2CA & RAC1 \\
YWHAQ & CREB3L2 & PIK3CA & EPOR \\
PPP2R2C & AKT3 & PIK3R1 & \\
\hline
\end{tabular}

Figure 6. PIK3CA is a direct target of miR-320a. (A) miR-320a target genes were predicted using TargetScan, miRwalk and miRDIP bioinformatics tools. (B) Enriched KEGG pathways of target genes of miR-320a. (C) Predicted miR-320a binding site in the 3'-UTR region of PIK3CA. (D) Predicted genes of the PI3K/Akt pathway. PIK3CA, phosphoinositide-3-kinase catalytic $\alpha$ polypeptide gene; miR, microRNA; miRDIP, microRNA Data Integration Portal; KEGG, Kyoto Encyclopedia of Genes and Genomes; UTR, untranslated region.

A

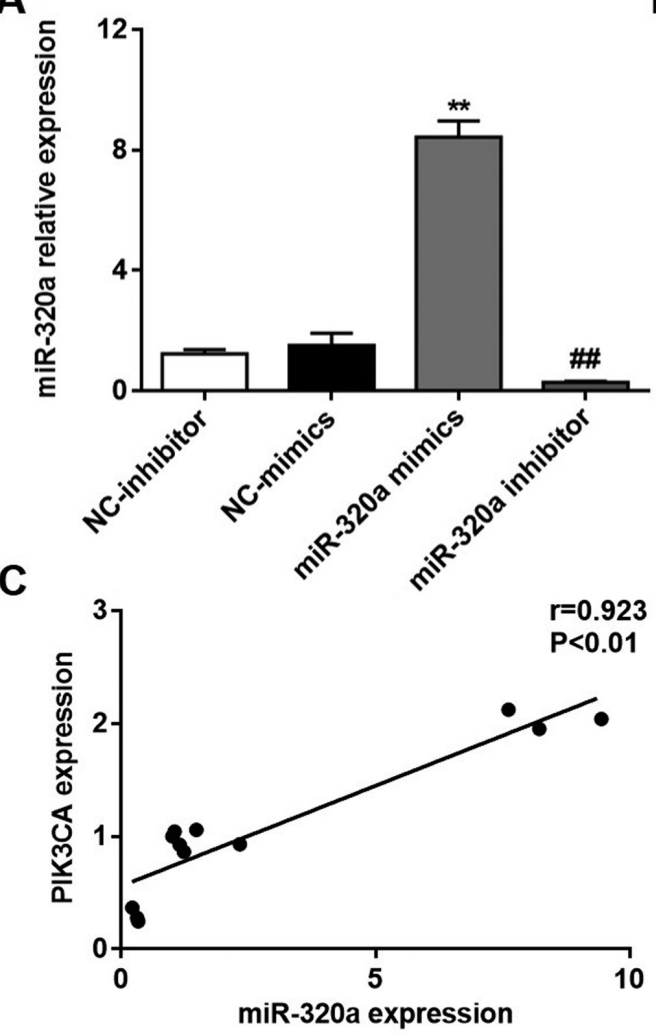

B

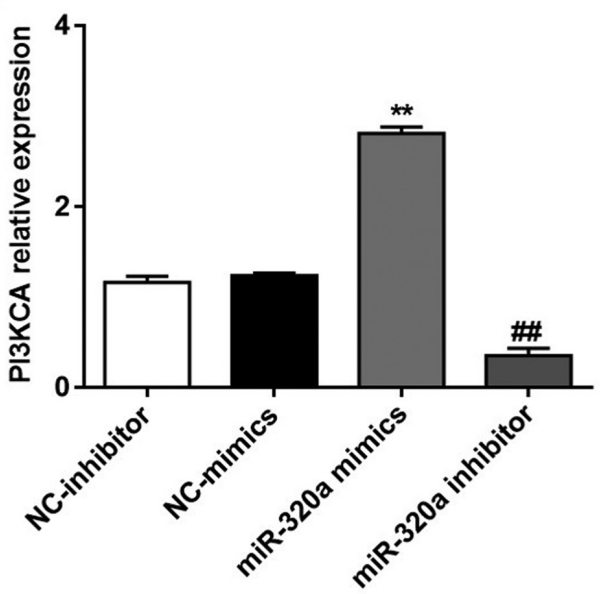

Figure 7. Expression of miR-320a and PIK3CA in HEH2 cells. Cells were seeded into 6-well plates and transfected with NC-mimics, NC-inhibitor, miR-320a mimics or miR-320a inhibitor for $72 \mathrm{~h}$. (A) miR-320a and (B) PIK3CA expression levels were quantified via reverse transcription-quantitative PCR. (C) Pearson's correlation analysis was performed to assess the correlation between miR-320a and PIK3CA expression levels. Data are presented as the mean \pm SEM from at least three independent experiments. Comparisons between two groups were analyzed using the unpaired Student's t-test. ${ }^{* * *} \mathrm{P}<0.01 \mathrm{vs}$. NC-mimics; ${ }^{\# \#} \mathrm{P}<0.01$ vs. NC-inhibitor. miR, microRNA; PIK3CA, phosphoinositide-3-kinase catalytic $\alpha$ polypeptide gene; NC, negative control. 

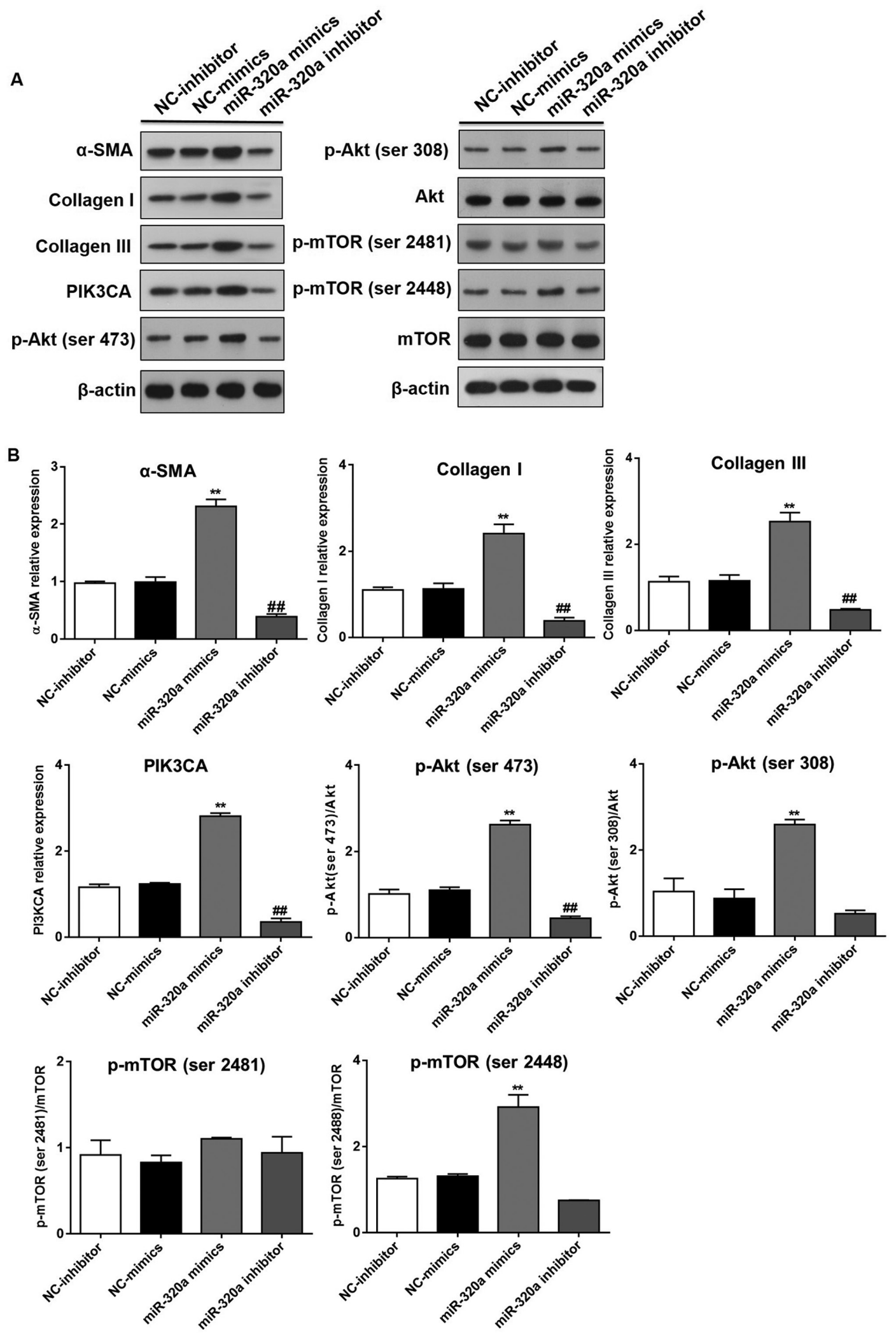

Figure 8. miR-320a regulates the PIK3CA/Akt/mTOR signaling pathway in HEH2 cells. Cells were seeded into 6-well plates and transfected with NC-mimics, NC-inhibitor, miR-320a mimics or miR-320a inhibitor for 72 h. $\alpha$-SMA, collagen I, collagen III, PIK3CA, p-Akt (ser 473), p-Akt (thr 308), Akt, p-mTOR (ser 2481), p-mTOR (ser 2488), mTOR and $\beta$-actin protein expression levels were (A) determined by western blotting and (B) semi-quantified. Data are presented as the mean \pm SEM from three independent experiments. Comparisons between two groups were analyzed using the unpaired Student's t-test. ${ }^{* *} \mathrm{P}<0.01$ vs. NC-mimics; ${ }^{\# \#} \mathrm{P}<0.01$ vs. NC-inhibitor. miR, microRNA; PIK3CA, phosphoinositide-3-kinase catalytic $\alpha$ polypeptide gene; NC, negative control; $\alpha$-SMA, $\alpha$-smooth muscle actin; p, phosphorylated.

the correlation between serum miR-320a and CHF, the key biomarkers for the diagnostic evaluation and risk stratification of patients with CHF, including LVEF, LVEDD, sST2, NT-proBNP and NYHA classification, were also evaluated. 
LVEF is the measurement of the volume of blood is being pumped out of the left ventricle of the heart. Patients with an $\mathrm{LVEF} \leq 40 \%$ can be categorized as experiencing heart failure (51). Furthermore, LVEDD is a predictive factor for mortality from heart failure and is closely associated with myocardial fibroblast proliferation (40). sST2 is the circulating form of the IL-33 receptor and serves an important role in the pathology of CHF (52). NT-proBNP is secreted by myocytes as a reaction to various stimuli, and the serum concentration of NT-proBNP is consistent with the clinical severity of CHF, serving as a sensitive marker of CHF (53). The present study demonstrated that serum miR-320a expression was positively correlated with sST2, LVEDD, N-terminal pro-BNP and NYHA classification, but negatively correlated with LVEF. These results suggested that miR-320a may be a risk factor for patients with CHF. Furthermore, circulating miRNAs exist in two distinct forms, including free and exosomal miRNAs (54). Previous studies have demonstrated that exosomal miRNAs mediated multiple intracellular signaling pathways and served an important role in cardiac fibroblasts in the pathogenesis of various diseases (55-57). To clarify whether miR-320a was transported by exosomes, exosomes were successfully extracted from serum samples of patients with $\mathrm{CHF}$ and healthy controls. The expression of miR-320a in exosomes isolated from patients with $\mathrm{CHF}$ was significantly elevated compared with the healthy control group. Furthermore, miR-320a was more abundant in serum exosomes compared with exosome-depleted supernatants (residues), indicating that exosomes were the primary carriers of miR-320a in serum. Therefore, exosomal miR-320a might be a useful diagnostic factor for patients with CHF.

Circulating exosomes transport miRNAs to the target organs, serve an important role in disease progression and facilitate myocardial fibroblast proliferation in CHF (58). The present study indicated that exosomes transported miR-320a to myocardial fibroblasts (HEH2 cells), indicating the potential of exosomal miR-320a to regulate myocardial fibrosis progression. To verify this hypothesis, miR-320a mimics and inhibitors were transfected into HEH2 cells. It has been reported that myocardial fibroblast proliferation is a determining factor in maintaining cardiac extracellular matrix and fibrotic myocardial remodeling in CHF, thus inhibiting this proliferation may serve as a reliable strategy to improve CHF (59). The results of the present study demonstrated that miR-320a knockdown significantly decreased cell viability and increased apoptotic rates in $\mathrm{HEH} 2$ cells compared with the $\mathrm{NC}$-inhibitor group. As one of the most specific parameters for CHF detection, sST2 is closely associated with myocardial fibrosis and ventricular remodeling (60). In the present study, compared with the corresponding NC groups, intracellular sST2 content was significantly increased following miR-320a mimics transfection, but decreased following miR-320a inhibitor transfection. These results suggested that miR-320a may promote proliferation of $\mathrm{HEH} 2$ cells. To elucidate the mechanisms underlying miR-320a, TargetScan, miRwalk and miRDIP were used to predict the biological target genes of miR-320a. The results revealed that PIK3CA, the gene encoding the p110 $\alpha$ subunit of PI3K, was one of the target genes of miR-320a. Compared with the NC-mimics group, miR-320a overexpression significantly upregulated the mRNA and protein expression levels of PIK3CA in HEH2 cells, which partially confirmed the prediction. Furthermore, KEGG analysis was performed to predict the associated signaling pathways. The results indicated that the PI3K/Akt pathway, which serves an important role in controlling myocardial fibrosis $(61,62)$, was one of the targets of miR-320a. Therefore, the potential downstream proteins that may participate in the miR-320a/PIK3CA axis in HEH2 cells were investigated in the present study. mTOR is a key regulator of protein synthesis in cardiomyocytes (63), serving an essential role in various cardiac diseases accompanied by cardiac fibrosis via promoting collagen expression, apoptosis, inflammation and autophagy $(64,65)$. As an upstream positive regulator of mTOR, Akt activates mTOR via a TSC complex subunit 2-independent mechanism by phosphorylating its four residues (66). In the present study, miR-320a mimics significantly upregulated the ratios of p-mTOR (ser 2448)/mTOR, p-Akt (ser 473)/Akt and p-Akt (ser 308)/Akt compared with the NC-mimics group. Furthermore, compared with the NC-inhibitor group, miR-320a inhibitor notably downregulated p-mTOR (ser 2448)/mTOR, p-Akt (ser 473)/Akt and p-Akt (ser 308)/Akt ratios in $\mathrm{HEH} 2$ cells, indicating that miR-320a promoted Akt/mTOR signal transduction. Previous studies have reported that the markers of myofibroblast proliferation, including $\alpha$-SMA, collagen I and collagen III $(67,68)$, were the downstream proteins of mTOR. The results of the present study suggested that compared with the corresponding $\mathrm{NC}$ groups, the expression levels of these three proteins were significantly upregulated following miR-320 mimics transfection, but significantly decreased following miR-320 inhibitor transfection, suggesting that miR-320 overexpression facilitated myocardial fibroblast proliferation via the PIK3CA/Akt/mTOR signaling pathway.

The present study had three primary limitations. First, the present study only investigated the expression of miR-320a in patients with $\mathrm{CHF}$ and demonstrated its effects on the PIK3CA/AKT/mTOR pathway in HEH2 cells. Future studies should employ other approaches to verify the effects and roles of miR-320a in CHF and myocardial fibroblast proliferation. Secondly, the present study only detected the percentage of apoptotic cells via flow cytometry. To verify the results of the present study, caspase signaling should be assessed to further determine the apoptotic effect of miR-320a. Thirdly, the effects of the mimics or inhibitor used in the present study may have been too strong to accurately represent the physiological effects of miR-320a. Therefore, future studies should use exosomes isolated from patients with $\mathrm{CHF}$ to determine whether exosomes display a similar effect to the mimics and inhibitor used in the present study.

In summary, the present study demonstrated that the expression of miR-320a was significantly higher in serum and exosomes isolated from patients with CHF compared with healthy controls. The results also indicated that exosomes were the primary carriers of miR-320a. Furthermore, compared with the NC-inhibitor group, miR-320a inhibitor transfection significantly decreased HEH2 cell viability and sST2 serum levels, and significantly increased apoptosis. The promotive effect of miR-320a on myocardial fibroblast proliferation may be associated with regulation of the PIK3CA/Akt/mTOR signaling pathway. Therefore, the results of the present study suggested 
that miR-320a may serve as a molecular target for the development of diagnostic and therapeutic strategies for $\mathrm{CHF}$.

\section{Acknowledgements}

Not applicable.

\section{Funding}

The present study was supported by the Construction Project of First-class Disciplines of Guang Xi (grant no. 2018XK070), the Fund of Key Subjects of Guang Xi Universities (grant no. 2017XK03) and Young Scientist Program of Beijing University of Chinese Medicine.

\section{Availability of data and materials}

The datasets used and/or analyzed during the current study are available from the corresponding author on reasonable request.

\section{Authors' contributions}

QGW and YZ conceived and designed the study. GL, SL, and LW designed the methodology. YZH, LJL, YL and YZ performed statistical analysis. QGW, YZH, LJL, YL and SL performed the experiments. YZH, BCYC and LJL analyzed the data. YZ and QGW wrote the original manuscript. BCYC revised the manuscript. QGW and YZ confirm the authenticity of all the raw data. All authors read and approved the final manuscript.

\section{Ethics approval and consent to participate}

The present study was reviewed and approved by the Institutional Review Board of the First Affiliated Hospital of Guang Xi University of Chinese Medicine [approval no. (2015)012].

\section{Patient consent for publication}

Not applicable.

\section{Competing interests}

The authors declare that they have no competing interests.

\section{References}

1. Heart failure...what of the future?. AIHW Bull 19: 2003.

2. Sochalski J, Jaarsma T, Krumholz HM, Laramee A, McMurray JJV, Naylor MD, Rich MW, Riegel B and Stewart S: What works in chronic care management: The case of heart failure. Health Aff (Millwood) 28: 179-189, 2009.

3. Azevedo PS, Polegato BF, Minicucci MF, Paiva SA and Zornoff LA: Cardiac remodeling: Concepts, clinical impact, pathophysiological mechanisms and pharmacologic treatment. Arq Bras Cardiol 106: 62-69, 2016 (In English, Portuguese).

4. Konstam MA, Kramer DG, Patel AR, Maron MS and Udelson JE: Left ventricular remodeling in heart failure: Current concepts in clinical significance and assessment. JACC Cardiovasc Imaging 4: 98-108, 2011.

5. Masci PG, Schuurman R, Andrea B, Ripoli A, Coceani M, Chiappino S, Todiere G, Srebot V, Passino C, Aquaro GD, et al: Myocardial fibrosis as a key determinant of left ventricular remodeling in idiopathic dilated cardiomyopathy: A contrast-enhanced cardiovascular magnetic study. Circ Cardiovasc Imaging 6: 790-799, 2013.
6. Xu F, Na L, Li Y and Chen L: Roles of the PI3K/AKT/mTOR signalling pathways in neurodegenerative diseases and tumours. Cell Biosci 10: 54, 2020.

7. Zhang Y, Zhou ZW, Jin H, Hu C, He ZX, Yu ZL, Ko KM, Yang T, Zhang X, Pan SY and Zhou SF: Schisandrin B inhibits cell growth and induces cellular apoptosis and autophagy in mouse hepatocytes and macrophages: Implications for its hepatotoxicity. Drug Des Devel Ther 9: 2001-2027, 2015.

8. Yang W, Wu Z, Yang K, Han Y, Chen Y, Zhao W, Huang F, Jin Y and Jin W: BMI1 promotes cardiac fibrosis in ischemia-induced heart failure via the PTEN-PI3K/Akt-mTOR signaling pathway. Am J Physiol Heart Circ Physiol 316: H61-H69, 2019.

9. Singla DK: Akt-mTOR pathway inhibits apoptosis and fibrosis in doxorubicin-induced cardiotoxicity following embryonic stem cell transplantation. Cell Transplant 24: 1031-1042, 2015.

10. Leask A: TGFbeta, cardiac fibroblasts, and the fibrotic response. Cardiovasc Res 74: 207-212, 2007.

11. Hu X, Zhang H, Li X, Li Y and Chen Z: Activation of mTORC1 in fibroblasts accelerates wound healing and induces fibrosis in mice. Wound Repair Regen 28: 6-15, 2020.

12. Macfarlane LA and Murphy PR: MicroRNA: Biogenesis, function and role in cancer. Curr Genomics 11: 537-561, 2010.

13. Osada $\mathrm{H}$ and Takahashi T: MicroRNAs in biological processes and carcinogenesis. Carcinogenesis 28: 2-12, 2007.

14. Shivdasani RA: MicroRNAs: Regulators of gene expression and cell differentiation. Blood 108: 3646-3653, 2006.

15. Bushati N and Cohen SM: microRNA functions. Annu Rev Cell Dev Biol 23: 175-205, 2007.

16. Yuan J, Liu H, Gao W, Zhang L, Ye Y, Yuan L, Ding Z, Wu J, Kang L, Zhang X, et al: MicroRNA-378 suppresses myocardial fibrosis through a paracrine mechanism at the early stage of cardiac hypertrophy following mechanical stress. Theranostics 8: 2565-2582, 2018.

17. Verjans R, Peters $T$, Beaumont FJ, van Leeuwen $R$, van Herwaarden T, Verhesen W, Munts C, Bijnen M, Henkens M, Diez J, et al: MicroRNA-221/222 family counteracts myocardial fibrosis in pressure overload-induced heart failure. Hypertension 71: 280-288, 2018.

18. Pegtel DM and Gould SJ: Exosomes. Annu Rev Biochem 88: 487-514, 2019.

19. Edgar JR: Q\&A: What are exosomes, exactly? BMC Biol 14: 46, 2016.

20. Bhome R, Del Vecchio F, Lee GH, Bullock MD, Primrose JN, Sayan AE and Mirnezami AH: Exosomal microRNAs (exomiRs): Small molecules with a big role in cancer. Cancer Lett 420: 228-235, 2018.

21. Matsumura T, Sugimachi K, Iinuma H, Takahashi Y, Kurashige J, Sawada G, Ueda M, Uchi R, Ueo H, Takano Y, et al: Exosomal microRNA in serum is a novel biomarker of recurrence in human colorectal cancer. Br J Cancer 113: 275-281, 2015.

22. Chen JJ, Zhao B, Zhao J and Li S: Potential roles of exosomal MicroRNAs as diagnostic biomarkers and therapeutic application in Alzheimer's disease. Neural Plast 2017: 7027380, 2017.

23. Yang VK, Loughran KA, Meola DM, Juhr CM, Thane KE, Davis AM and Hoffman AM: Circulating exosome microRNA associated with heart failure secondary to myxomatous mitral valve disease in a naturally occurring canine model. J Extracell Vesicles 6: 1350088, 2017.

24. Tian ZQ, Jiang H and Lu ZB: MiR-320 regulates cardiomyocyte apoptosis induced by ischemia-reperfusion injury by targeting AKIP1. Cell Mol Biol Lett 23: 41, 2018.

25. Ren XP, Wu J, Wang X, Sartor MA, Jones K, Qian J, Nicolaou P, Pritchard TJ and Fan GC: MicroRNA-320 is involved in the regulation of cardiac ischemia/reperfusion injury by targeting heat-shock protein 20. Circulation 119: 2357-2366, 2009.

26. Chinese Society of Cardiology of Chinese Medical Association; Editorial Board of Chinese Journal of Cardiology: Chinese guidelines for the diagnosis and treatment of heart failure 2014. Zhonghua Xin Xue Guan Bing Za Zhi 42: 98-122, 2014 (In Chinese).

27. Caraballo C, Desai NR, Mulder H, Alhanti B, Wilson FP, Fiuzat M, Felker GM, Piña IL, O'Connor CM, Lindenfeld J, et al: Clinical implications of the New York heart association classification. J Am Heart Assoc 8: e014240, 2019.

28. Zhang Y, Yan LS, Ding Y, Cheng BCY, Luo G, Kong J, Liu TH and Zhang SF: Edgeworthia gardneri (Wall.) meisn. Water extract ameliorates palmitate induced insulin resistance by regulating IRS1/GSK3 $\beta /$ FoxO1 signaling pathway in human HepG2 hepatocytes. Front Pharmacol 10: 1666, 2020. 
29. Barrett T, Wilhite SE, Ledoux P, Evangelista C, Kim IF, Tomashevsky M, Marshall KA, Phillippy KH, Sherman PM, Holko M, et al: NCBI GEO: Archive for functional genomics data sets-update. Nucleic Acids Res 41: D991-D995, 2013.

30. Huang Z, Shi J, Gao Y, Cui C, Zhang S, Li J, Zhou Y and Cui Q: HMDD v3.0: A database for experimentally supported human microRNA-disease associations. Nucleic Acids Res 47: D1013-D1017, 2019.

31. Agarwal V, Bell GW, Nam J and Bartel DP: Predicting effective microRNA target sites in mammalian mRNAs. ELife 4: e05005, 2015.

32. Dweep H, Sticht C, Pandey P and Gretz N: miRWalk-database: Prediction of possible miRNA binding sites by 'walking' the genes of three genomes. J Biomed Inform 44: 839-847, 2011

33. Tokar T, Pastrello C, Rossos AEM, Abovsky M, Hauschild AC, Tsay M, Lu R and Jurisica I: mirDIP 4.1-integrative database of human microRNA target predictions. Nucleic Acids Res 46 D360-D370, 2018

34. Huang DW, Sherman BT and Lempicki RA: Bioinformatics enrichment tools: Paths toward the comprehensive functional analysis of large gene lists. Nucleic Acids Res 37: 1-13, 2009.

35. Kanehisa M, Furumichi M, Sato Y, Ishiguro-Watanabe $M$ and Tanabe M: KEGG: Integrating viruses and cellular organisms. Nucleic Acids Res 49: D545-D551, 2021.

36. Livak KJ and Schmittgen TD: Analysis of relative gene expression data using real-time quantitative PCR and the 2(-Delta Delta C(T)) method. Methods 25: 402-408, 2001

37. Villacorta $\mathrm{H}$ and Maisel AS: Soluble ST2 testing: A promising biomarker in the management of heart failure. Arq Bras Cardiol 106: 145-152, 2016 (In English, Portuguese).

38. Wojtczak-Soska K, Pietrucha T, Sakowicz A and Lelonek M: Soluble ST2 protein in chronic heart failure is independent of traditional factors. Arch Med Sci 9: 21-26, 2013

39. Tsuji K, Sakata Y, Nochioka K, Miura M, Yamauchi T, Onose T, Abe R, Oikawa T, Kasahara S, Sato M, et al: Characterization of heart failure patients with mid-range left ventricular ejection fraction-a report from the CHART-2 Study. Eur J Heart Fail 19: $1258-1269,2017$

40. Holland R, Rechel B, Stepien K, Harvey I and Brooksby I Patients' self-assessed functional status in heart failure by New York heart association class: A prognostic predictor of hospitalizations, quality of life and death. J Card Fail 16: 150-156, 2010

41. Glaubitz M, Block S, Witte J, Empen K, Gross S, Schlicht R Weitmann K, Klingel K, Kandolf R, Hoffmann W, et al: Stiffness of left ventricular cardiac fibroblasts is associated with ventricular dilation in patients with recent-onset nonischemic and nonvalvular cardiomyopathy. Circ J 78: 1693-1700, 2014

42. Kumar D, Gupta D, Shankar S and Srivastava RK: Biomolecular characterization of exosomes released from cancer stem cells: Possible implications for biomarker and treatment of cancer. Oncotarget 6: 3280-3291, 2015

43. Puzar Dominkus P, Stenovec M, Sitar S, Lasič E, Zorec R, Plemenitaš A, Žagar E, Kreft M and Lenassi M: PKH26 labeling of extracellular vesicles: Characterization and cellular internalization of contaminating PKH26 nanoparticles. Biochim Biophys Acta Biomembr 1860: 1350-1361, 2018.

44. Fan H, Ma L, Fan B, Wu J, Yang Z and Wang L: Role of PDGFR- $\beta / P I 3 K / A K T$ signaling pathway in PDGF-BB induced myocardial fibrosis in rats. Am J Transl Res 6: 714-723, 2014

45. Willis O, Choucair K, Alloghbi A, Stanbery L, Mowat R, Brunicardi FC, Dworkin L and Nemunaitis J: PIK3CA gene aberrancy and role in targeted therapy of solid malignancies. Cancer Gene Ther 27: 634-644, 2020.

46. Lieb V, Weigelt K, Scheinost L, Fischer K, Greither T, Marcou M, Theil G, Klocker H, Holzhausen HJ, Lai X, et al: Serum levels of miR-320 family members are associated with clinical parameters and diagnosis in prostate cancer patients. Oncotarget 9: 10402-10416, 2017.

47. Chen C, Wang Y, Yang S, Li H, Zhao G, Wang F, Yang L and Wang DW: MiR-320a contributes to atherogenesis by augmenting multiple risk factors and down-regulating SRF. J Cell Mol Med 19: 970-985, 2015

48. Yin Z, Zhao Y, Li H, Yan M, Zhou L, Chen C and Wang DW: miR-320a mediates doxorubicin-induced cardiotoxicity by targeting VEGF signal pathway. Aging (Albany NY) 8: 192-207, 2016.

49. Sommariva E, D'Alessandra Y, Farina FM, Casella M, Cattaneo F, Catto V, Chiesa M, Stadiotti I, Brambilla S, Russo AD, et al: MiR-320a as a potential novel circulating biomarker of arrhythmogenic cardiomyopathy. Sci Rep 7: 4802, 2017.
50. Salloum-Asfar S, Satheesh NJ and Abdulla SA: Circulating miRNAs, small but promising biomarkers for autism spectrum disorder. Front Mol Neurosci 12: 253, 2019.

51. Ponikowski P, Voors AA, Anker SD, Bueno H, Cleland JG, Coats AJ, Falk V, González-Juanatey JR, Harjola VP, Jankowska EA, et al: 2016 ESC guidelines for the diagnosis and treatment of acute and chronic heart failure: The task force for the diagnosis and treatment of acute and chronic heart failure of the European society of cardiology (ESC) developed with the special contribution of the heart failure association (HFA) of the ESC. Eur Heart J 37: 2129-2200, 2016.

52. Lotierzo M, Dupuy AM, Kalmanovich E, Roubille F and Cristol JP: sST2 as a value-added biomarker in heart failure. Clin Chim Acta 501: 120-130, 2020.

53. Ozturk TC, Unluer E, Denizbasi A, Guneysel O and Onur O: Can NT-proBNP be used as a criterion for heart failure hospitalization in emergency room?. J Res Med Sci 16: 1564-1571, 2011.

54. Turchinovich A, Weiz L, Langheinz A and Burwinkel B Characterization of extracellular circulating microRNA. Nucleic Acids Res 39: 7223-7233, 2011

55. Wang B, Zhang A, Wang H, Klein JD, Tan L, Wang ZM, Du J, Naqvi N, Liu BC and Wang XH: miR-26a limits muscle wasting and cardiac fibrosis through exosome-mediated microRNA transfer in chronic kidney disease. Theranostics 9: 1864-1877, 2019.

56. Yang J, Yu X, Xue F, Li Y, Liu W and Zhang S: Exosomes derived from cardiomyocytes promote cardiac fibrosis via myocyte-fibroblast cross-talk. Am J Transl Res 10: 4350-4366, 2018.

57. Bang C, Batkai S, Dangwal S, Gupta SK, Foinquinos A, Holzmann A, Just A, Remke J, Zimmer K, Zeug A, et al: Cardiac fibroblast-derived microRNA passenger strand-enriched exosomes mediate cardiomyocyte hypertrophy. J Clin Invest 124: 2136-2146, 2014

58. Wang L, Liu J, Xu B, Liu YL and Liu Z: Reduced exosome miR-425 and miR-744 in the plasma represents the progression of fibrosis and heart failure. Kaohsiung J Med Sci 34: 626-633, 2018.

59. Lv T, Du Y, Cao N, Zhang S, Gong Y, Bai Y, Wang W and Liu H: Proliferation in cardiac fibroblasts induced by $\beta 1$-adrenoceptor autoantibody and the underlying mechanisms. Sci Rep 6: 32430, 2016.

60. Januzzi JL, Mebazaa A and Di Somma S: ST2 and prognosis in acutely decompensated heart failure: The international ST2 consensus panel. Am J Cardiol 115 (Suppl 7): 26B-31B, 2015

61. Ock S, Lee WS, Ahn J, Kim HM, Kang H, Kim HS, Jo D, Abel ED, Lee TJ and Kim J: Deletion of IGF-1 receptors in cardiomyocytes attenuates cardiac aging in male mice. Endocrinology 157 : 336-345, 2016.

62. Wang WW, Zhang FL, Chen JH, Chen XH, Fu FY, Tang MR and Chen LL: Telmisartan reduces atrial arrhythmia susceptibility through the regulation of RAS-ERK and PI3K-Akt-eNOS pathways in spontaneously hypertensive rats. Can J Physio Pharmacol 93: 657-665, 2015.

63. McMullen JR, Sherwood MC, Tarnavski O, Zhang L, Dorfman AL, Shioi T and Izumo S: Inhibition of mTOR signaling with rapamycin regresses established cardiac hypertrophy induced by pressure overload. Circulation 109: 3050-3055, 2004

64. Sun S, Hu Y, Zheng Q, Guo Z, Sun D, Chen S, Zhang Y, Liu P, $\mathrm{Lu} \mathrm{J}$ and Jiang J: Poly(ADP-ribose) polymerase 1 induces cardiac fibrosis by mediating mammalian target of rapamycin activity. J Cell Biochem 120: 4813-4826, 2019.

65. Laplante M and Sabatini DM: mTOR signaling at a glance. J Cell Sci 122: 3589-3594, 2009

66. Memmott RM and Dennis PA: Akt-dependent and -independent mechanisms of mTOR regulation in cancer. Cell Signal 21: 656-664, 2009

67. Rao KB, Malathi N, Narashiman S and Rajan ST: Evaluation of myofibroblasts by expression of alpha smooth muscle actin: A marker in fibrosis, dysplasia and carcinoma. J Clin Diagn Res 8: ZC14-ZC17, 2014.

68. Zhang K, Rekhter MD, Gordon D and Phan SH: Myofibroblasts and their role in lung collagen gene expression during pulmonary fibrosis. A combined immunohistochemical and in situ hybridization study. Am J Pathol 145: 114-125, 1994.

This work is licensed under a Creative Commons Attribution-NonCommercial-NoDerivatives 4.0 International (CC BY-NC-ND 4.0) License. 